\title{
Collective excitation frequencies and stationary states of trapped dipolar Bose-Einstein condensates in the Thomas-Fermi regime
}

\author{
R. M. W. van Bijnen, ${ }^{1,2}$ N. G. Parker, ${ }^{2,3}$ S. J. J. M. F. Kokkelmans, ${ }^{1}$ A. M. Martin, ${ }^{4}$ and D. H. J. O'Dell ${ }^{2}$ \\ ${ }^{1}$ Eindhoven University of Technology, P.O. Box 513, NE-5600 MB Eindhoven, The Netherlands \\ ${ }^{2}$ Department of Physics and Astronomy, McMaster University, Hamilton, Ontario L8S 4M1, Canada \\ ${ }^{3}$ School of Food Science and Nutrition, University of Leeds, Leeds LS2 9JT, United Kingdom \\ ${ }^{4}$ School of Physics, University of Melbourne, Parkville, Victoria 3010, Australia
}

(Received 28 February 2010; published 15 September 2010)

\begin{abstract}
We present a general method for obtaining the exact static solutions and collective excitation frequencies of a trapped Bose-Einstein condensate (BEC) with dipolar atomic interactions in the Thomas-Fermi regime. The method incorporates analytic expressions for the dipolar potential of an arbitrary polynomial density profile, thereby reducing the problem of handling nonlocal dipolar interactions to the solution of algebraic equations. We comprehensively map out the static solutions and excitation modes, including non-cylindrically-symmetric traps, and also the case of negative scattering length where dipolar interactions stabilize an otherwise unstable condensate. The dynamical stability of the excitation modes gives insight into the onset of collapse of a dipolar BEC. We find that global collapse is consistently mediated by an anisotropic quadrupolar collective mode, although there are two trapping regimes in which the BEC is stable against quadrupole fluctuations even as the ratio of the dipolar to $s$-wave interactions becomes infinite. Motivated by the possibility of a fragmented condensate in a dipolar Bose gas due to the partially attractive interactions, we pay special attention to the scissors modes, which can provide a signature of superfluidity, and identify a long-range restoring force which is peculiar to dipolar systems. As part of the supporting material for this paper we provide the computer program used to make the calculations, including a graphical user interface.
\end{abstract}

DOI: 10.1103/PhysRevA.82.033612

PACS number(s): 03.75.Kk, 34.20.Cf

\section{INTRODUCTION}

Since the realization of atomic Bose-Einstein condensates (BECs) in 1995 [1], there has been a surge of interest in quantum degenerate gases [2,3]. Despite the diluteness of these gases, interatomic interactions play an important role in determining their properties. In the majority of experiments, the dominant interactions have been isotropic and asymptotically of the van der Waals type, falling off as $1 / r^{6}$. At ultracold temperatures this leads to essentially pure $s$-wave scattering between the atoms. An exception to this rule is provided by gases that have significant dipole-dipole interactions [4-7]. In comparison to van der Waals type interactions, dipolar interactions are longer range and anisotropic, and this introduces rich, new phenomena. For example, a series of experiments that have revealed the anisotropic nature of dipolar interactions are those on ${ }^{52} \mathrm{Cr}$ BECs in an external magnetic field. These have demonstrated anisotropic expansion of the condensate depending on the direction of polarization of the atomic dipoles [8,9], collapse and $d$-wave explosion [10], and an enhanced stability against collapse in flattened geometries [11]. Meanwhile, an experiment with ${ }^{39} \mathrm{~K}$ atoms occupying different sites in a one-dimensional optical lattice has demonstrated the long-range nature of dipolar interactions in BECs through dephasing of Bloch oscillations [6]. Dipolar interactions have also been shown to be responsible for the formation of a spatially modulated structure of spin domains in a ${ }^{87} \mathrm{Rb}$ spinor BEC [7].

In order to incorporate atomic interactions into the GrossPitaevskii theory for the condensate one should use a pseudopotential [2,3]. In the presence of both dipolar and van der Waals interactions the pseudopotential can be written as the sum of two terms $U(\mathbf{r})=U_{s}(\mathbf{r})+U_{\mathrm{dd}}(\mathbf{r})$ [12-15], where $\mathbf{r}$ is the relative interatomic separation. The long-range dipolar interaction can be treated accurately within the Born approximation providing one is not close to a scattering resonance $[14,15]$. This first-order approximation means that the effective interaction is replaced by the potential itself. This is quite different from the shorter-range van der Waals interaction, for which the Born approximation is not valid at low temperatures, and where one rather uses the contact potential

$$
U_{s}(\mathbf{r})=g \delta(\mathbf{r}) .
$$

The $s$-wave coupling constant $g=4 \pi \hbar^{2} a_{\mathrm{s}} / m$ is given in terms of the scattering length $a_{\mathrm{s}}$ and the atomic mass $m$. For the dipolar interaction, we consider two atoms whose dipoles are aligned by an external field pointing along the direction specified by the unit vector $\hat{\mathbf{e}}$. The potential is then given by

$$
U_{\mathrm{dd}}(\mathbf{r})=\frac{C_{\mathrm{dd}}}{4 \pi} \hat{e}_{i} \hat{e}_{j} \frac{\left(\delta_{i j}-3 \hat{r}_{i} \hat{r}_{j}\right)}{r^{3}},
$$

where $C_{\mathrm{dd}}$ parametrizes the strength of the dipolar interactions, $\hat{\mathbf{r}}$ is a unit vector in the direction of $\mathbf{r}$, and summation over repeated indices is implied. A key figure of merit is the ratio of the two coupling strengths, defined as [16]

$$
\varepsilon_{\mathrm{dd}}=C_{\mathrm{dd}} / 3 g \text {. }
$$

Dipole-dipole interactions can be either magnetic or electric in origin. To date, the dipolar interactions seen in ultracold atom experiments [4-7] have all been magnetic dipolar interactions, for which $C_{\mathrm{dd}}=\mu_{0} d^{2}$, where $d$ is the magnetic dipole moment and $\mu_{0}$ is the permeability of free space. In terms of the Bohr magneton $\mu_{\mathrm{B}}$, the magnetic dipole moment of a ${ }^{52} \mathrm{Cr}$ atom is $d=6 \mu_{\mathrm{B}}$ giving $\varepsilon_{\mathrm{dd}} \approx 0.16$ [4]. Although this is 36 times larger 
than the typical value of $\varepsilon_{\mathrm{dd}}$ found in the alkali-metal atoms, it is still small. Thus, unless the system is in a configuration that makes it particularly sensitive [6], and/or is specially prepared [7], the magnetic dipolar interactions in the atomic gases made so far tend to be masked by stronger $s$-wave interactions. In order to make dipolar interactions in BECs more visible, the Stuttgart group have succeeded in implementing magnetic Feshbach resonances [17] in ${ }^{52} \mathrm{Cr}[11,18]$. These allow $g$ to be tuned from positive to negative and even to zero. Moreover, the sign and amplitude of the effective value of $C_{\mathrm{dd}}$ can also be tuned by rapidly rotating the external polarizing field [16]. Polar molecules can have huge electric dipole moments, and these systems are now close to reaching degeneracy [19-24]. By appropriately tuning an external electric field, a large degree of control can be exerted over these systems [24]. Combined with what has already been achieved in ${ }^{52} \mathrm{Cr}$, a large parameter space of interactions can now be realistically explored in dipolar BECs.

The ground state of a trapped dipolar BEC has already been investigated theoretically by a number of authors, e.g., [12-15,25-32], with most studies focusing on the regime where $g \geqslant 0$ and $C_{\mathrm{dd}}>0$. The presence of dipolar interactions was widely predicted to lead to certain distinctive effects, some of which have recently been seen experimentally. For example, if the dipoles are aligned in the $z$ direction, then a condensate will elongate along $z$ and become more cigar shaped, i.e., undergo magnetostriction, in order to benefit energetically from the attractive end-to-end interaction of dipoles. As $\varepsilon_{\mathrm{dd}}$ is increased, for example, by reducing $g$ with a Feshbach resonance, the BEC eventually becomes unstable to collapse, and this striking behavior has been realized in the experiment [10]. Conversely, a condensate that is flattened by strong trapping along $z$ will be mostly composed of repulsive side-by-side dipoles, and so this pancake-shaped geometry is more stable, as confirmed experimentally [11]. In the limit that $\varepsilon_{\mathrm{dd}}$ becomes large but the BEC remains in the pancake configuration due to tight trapping, remarkable density wave structures have been predicted for certain regions of parameter space close to the collapse threshold [29-31].

In this paper we work in the Thomas-Fermi (TF) regime, which is of rather general interest because it is formally equivalent to the hydrodynamic regime of zero-temperature superfluids [33]. The TF regime may be viewed as the semiclassical approximation to the full Gross-Pitaevskii theory. A stationary condensate enters the TF regime when the zeropoint kinetic energy of the atoms due to the confinement by the trap becomes negligible compared to the total interaction and trapping energies. For BECs with repulsive interactions in a harmonic trap, this generally occurs in the large $N$ limit, where $N$ is the number of atoms. However, for dipolar BECs the picture is considerably complicated by the partially attractive and partially repulsive nature of the interactions. The question of the validity of the TF regime in static dipolar BECs has been addressed in [34]. The validity of results for collective excitations, which is the major theme of this paper, rests primarily on the validity of the underlying static solution about which they are a perturbation (we are interested in small amplitude excitations here). Theoretical results [32] demonstrate that if the static solution closely approximates the true Gross-Pitaevskii solution, then the TF equations of motion (the superfluid hydrodynamic equations) give a remarkably robust account of the dynamics. For example, excellent agreement was observed between the TF model and full simulations of the Gross-Pitaevskii equation for a dipolar BEC even for extreme perturbations to the condensate, including the initial dynamics of collapse. Thus, for the small perturbations considered here, we can be confident of consistent results, providing the underlying static solution is valid.

The TF regime is theoretically simpler to handle than the full Gross-Pitaevskii theory, thereby facilitating analytical results. For example, under harmonic trapping it can be shown that the exact density profile of a dipolar condensate in the TF regime is an inverted parabola [26,27], similar to the usual $s$-wave case but distorted by the magnetostriction. Furthermore, the stability of the ground state to collapse can be estimated simply in the TF regime and reasonable agreement with experiment has been reported [11]. Rotational instabilities of dipolar BECs are also amenable to analysis in the TF regime $[35,36]$. The current paper builds on these earlier works by applying the exact results available in the TF regime to collective excitations.

The excited states of a BEC can be accurately calculated within the TF regime provided they are of sufficiently long wavelength. The most basic collective excitations of a trapped BEC are the dipole (center-of-mass), monopole (breathing), quadrupole, and scissors modes, illustrated schematically in Fig. 1. Their characterization offers important opportunities for measuring interaction effects, testing theoretical models, and even detecting weak forces [37]. Specifically, the scissors mode provides an important test for superfluidity [38-41], while the quadrupole mode plays a key role in the onset of vortex nucleation in rotating condensates [35,36,42-46]. An instability of the quadrupole mode is also thought to be the mechanism by which collapse of dipolar BECs proceeds when it occurs globally [14,25,32,47] (rather than locally [32]). While the collective modes of a dipolar BEC have been studied
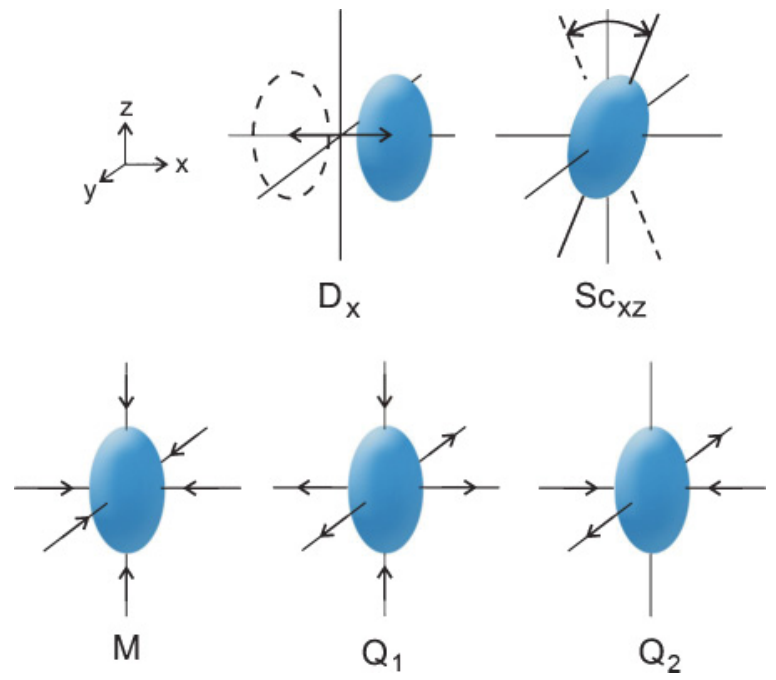

FIG. 1. (Color online) Schematic illustration of the basic collective modes under consideration: the dipole mode $D$ (shown here in the $x$ direction $D_{x}$ ), scissors mode $\mathrm{Sc}$ (shown here in $x-z$ plane $\mathrm{Sc}_{x z}$ ), the monopole mode $M$, and the quadrupole modes $Q_{1}$ and $Q_{2}$. These modes are discussed in more detail in Sec. III. 
previously [25,26,28,48-51], key issues remain at large, for example, the regimes of $C_{\mathrm{dd}}<0$ and $g<0$, and the behavior of the scissors modes. This provides the motivation for the current work.

In this paper we present a general and accessible methodology for determining the static solutions and excitation frequencies of trapped dipolar BECs in the TF limit. We explore the static solutions and low-lying collective excitations throughout a large and experimentally relevant parameter space, including positive and negative dipolar couplings $C_{\mathrm{dd}}$, positive and negative $s$-wave interactions $g$, and cylindricallyand non-cylindrically-symmetric systems. Moreover, our approach enables us to unambiguously identify the modes responsible for global collapse of the condensate. We would like to point out that there is a freely available MATLAB implementation of the calculations presented in this paper, complete with a graphical user interface, which can be found in the supporting material [52].

Section II is devoted to the static solutions of the system. Beginning with the underlying Gross-Pitaevskii theory for the condensate mean field, we make the TF approximation and outline the methodology for deriving the TF static solutions. We then use it to map out the static solutions with cylindrical symmetry, for both repulsive and attractive $s$-wave interactions, and then present an example case of the static solutions in a non-cylindrically-symmetric geometry. We compare to recent experimental observations where possible.

In Sec. III we present our methodology for deriving the excitation frequencies of a dipolar BEC. This is an adaption of the method that Sinha and Castin applied to standard $s$-wave condensates [42] where one considers perturbations around the static solutions (derived in Sec. II) and employs linearized equations of motion for these perturbations. At the heart of our approach is the exact calculation of the dipolar potential of a heterogeneous ellipsoidal BEC, performed by employing results from gravitational potential theory known in astrophysics [53-58] and detailed in Appendices B and C.

In Sec. IV we apply this method to calculate the frequencies of the important low-lying modes of the system, namely, the monopole, dipole, quadrupole, and scissors modes, for cylindrically symmetric traps. We show how these frequencies vary with the key parameters of the system, $\varepsilon_{\mathrm{dd}}$ and trap ratio $\gamma$, and give physical explanations for our observations. In Sec. V we extend our analysis to non-cylindrically-symmetric traps. Although the parameter space of such systems is very large, we present pertinent examples. An important feature of non-cylindrically-symmetric ground states is that they support a family of scissors modes which can be employed as a test for superfluidity. As such, in Sec. VI, we focus on these scissors modes and show how they vary with key parameters. Finally, in Sec. VII, we summarize our findings.

There are three appendices included in this paper. Appendix A contains a plot of the frequencies of the collective modes of the BEC as a function of $\varepsilon_{\mathrm{dd}}$. Appendix B outlines the method by which we calculate dipolar potentials due to arbitrary polynomial density distributions of atoms. This is the main technical advance of this work over our previous papers which were limited to the dipolar potentials associated with strictly paraboloidal density distributions, i.e., those of the same symmetry class as the static solution. In Appendix C we give a closed formula in terms of elliptic integrals for the dipolar potential inside a triaxial ellipsoid with a parabolic density profile. This is a special but important case of the general theory outlined in Appendix B.

\section{STATIC SOLUTIONS}

\section{A. Methodology for obtaining static solutions}

At zero temperature the condensate is well described by a mean-field order parameter, or "wave function," $\psi(\mathbf{r}, t)$. This defines an atomic density distribution via $n(\mathbf{r}, t)=|\psi(\mathbf{r}, t)|^{2}$. Static solutions, denoted by $\tilde{\psi}(\mathbf{r})$, satisfy the time-independent Gross-Pitaevskii equation (GPE) given by [3]

$$
\left(-\frac{\hbar^{2}}{2 m} \nabla^{2}+V(\mathbf{r})+\Phi_{\mathrm{dd}}(\mathbf{r})+g|\tilde{\psi}(\mathbf{r})|^{2}\right) \tilde{\psi}(\mathbf{r})=\mu \tilde{\psi}(\mathbf{r}),
$$

where $\mu$ is the chemical potential of the system. The external potential $V(\mathbf{r})$ is typically harmonic with the general form

$$
V(\mathbf{r})=\frac{1}{2} m \omega_{\perp}^{2}\left[(1-\epsilon) x^{2}+(1+\epsilon) y^{2}+\gamma^{2} z^{2}\right] .
$$

Here $\omega_{\perp}$ is the average trap frequency in the $x-y$ plane and the trap aspect ratio $\gamma=\omega_{z} / \omega_{\perp}$ defines the trapping in the axial (z) direction. The trap ellipticity $\epsilon$ in the $x-y$ plane defines the transverse trap frequencies via $\omega_{x}=\sqrt{1-\epsilon} \omega_{\perp}$ and $\omega_{y}=$ $\sqrt{1+\epsilon} \omega_{\perp}$. When $\epsilon=0$ the trap is cylindrically symmetric.

The $\Phi_{\mathrm{dd}}$ term in Eq. (4) is the mean-field potential arising from the dipolar interactions

$$
\Phi_{\mathrm{dd}}(\mathbf{r})=\int n\left(\mathbf{r}^{\prime}\right) U_{\mathrm{dd}}\left(\mathbf{r}-\mathbf{r}^{\prime}\right) d^{3} r^{\prime} .
$$

This term is a nonlocal functional of the density and is the source of the difficulties associated with theoretical treatments of dipolar BECs: it turns the GPE into an integro-differential equation. A key feature of the approach taken by us in this paper is to calculate this term analytically. To this end we express the dipolar mean field in terms of a fictitious electrostatic potential $\phi(\mathbf{r})[26,27,59]$

$$
\Phi_{\mathrm{dd}}(\mathbf{r})=-C_{\mathrm{dd}}\left(\frac{\partial^{2}}{\partial z^{2}} \phi(\mathbf{r})+\frac{1}{3} n(\mathbf{r})\right),
$$

where

$$
\phi(\mathbf{r})=\frac{1}{4 \pi} \int \frac{n\left(\mathbf{r}^{\prime}\right)}{\left|\mathbf{r}-\mathbf{r}^{\prime}\right|} d^{3} r^{\prime}
$$

$\phi(\mathbf{r})$ satisfies Poisson's equation $\nabla^{2} \phi(\mathbf{r})=-n(\mathbf{r})$. Note that in (7) we have taken the dipoles to be aligned along the $z$ direction. The term $n(\mathbf{r}) / 3$ appearing on the right-hand side of (7) cancels the Dirac $\delta$ function which arises in the $\partial^{2} \phi(\mathbf{r}) \partial z^{2}$ term $[59,60]$. This means that $\Phi_{\text {dd }}$ includes only the long-range $\left(r^{-3}\right)$ part of the dipolar interaction, exactly as written in Eq. (2).

We assume the TF approximation where the zero-point kinetic energy of the atoms in the trap is neglected. Dropping the relevant $\nabla^{2}$ term in Eq. (4) leads to

$$
V(\mathbf{r})+\Phi_{\mathrm{dd}}(\mathbf{r})+g n(\mathbf{r})=\mu .
$$

For an $s$-wave BEC under harmonic trapping, the exact density profile in the TF approximation is known to be an inverted 
parabola [3] with the general form

$$
n(\mathbf{r})=n_{0}\left(1-\frac{x^{2}}{R_{x}^{2}}-\frac{y^{2}}{R_{y}^{2}}-\frac{z^{2}}{R_{z}^{2}}\right) \text { for } n(\mathbf{r}) \geqslant 0,
$$

where $n_{0}=15 N /\left(8 \pi R_{x} R_{y} R_{z}\right)$ is the central density, and $R_{x}, R_{y}$, and $R_{z}$ are the condensate radii. In order to obtain the dipolar potential arising from this density distribution, one must find the corresponding electrostatic potential of Eq. (8). References [26,27] follow this procedure and arrive at the remarkable conclusion that the dipolar potential $\Phi_{\mathrm{dd}}$ is also parabolic. Therefore, a parabolic density profile is also an exact solution of the time-independent TF equation (9) even in the presence of dipolar interactions. In Sec. III and Appendices B and $\mathrm{C}$ we point out that this result can be extended using results from 19th-century gravitational potential theory [53,54,57] to arbitrary polynomial densities yielding polynomial dipolar potentials of the same degree. For the parabolic density profile at hand, the internal dipolar potential is given by $[27,35]$

$$
\begin{aligned}
\Phi_{\mathrm{dd}}(\mathbf{r})= & -g \varepsilon_{\mathrm{dd}} n(\mathbf{r})+\frac{3 g \varepsilon_{\mathrm{dd}} n_{0} \kappa_{x} \kappa_{y}}{2} \\
& \times\left[\beta_{001}-\left(\beta_{101} x^{2}+\beta_{011} y^{2}+3 \beta_{002} z^{2}\right) R_{z}^{-2}\right],
\end{aligned}
$$

where $\kappa_{x}=R_{x} / R_{z}$ and $\kappa_{y}=R_{y} / R_{z}$ are the aspect ratios of the condensate, and

$$
\beta_{i j k}=\int_{0}^{\infty} \frac{d s}{\left(\kappa_{x}^{2}+s\right)^{i+\frac{1}{2}}\left(\kappa_{y}^{2}+s\right)^{j+\frac{1}{2}}(1+s)^{k+\frac{1}{2}}},
$$

where $i, j, k$ are integers. Explicit expressions for $\beta_{001}, \beta_{101}, \beta_{011}$, and $\beta_{002}$ in terms of elliptic integrals are given in Appendix C. Note that for a cylindrically symmetric trap $\epsilon=0$, the static condensate profile is also cylindrically symmetric with aspect ratio $\kappa_{x}=\kappa_{y}=: \kappa$. In the cylindrically symmetric case the integrals $\beta_{i j k}$ of Eq. (12) can be evaluated in terms of the ${ }_{2} F_{1}$ Gauss hypergeometric function $[61,62]$ for any $i, j, k$

$$
\beta_{i j k}=2 \frac{{ }_{2} F_{1}\left(k+\frac{1}{2}, 1 ; i+j+k+\frac{3}{2} ; 1-\kappa^{2}\right)}{(1+2 i+2 j+2 k) \kappa^{2(i+j)}} .
$$

For the parabolic density profile of Eq. (10), the static TF Eq. (9) becomes

$$
\begin{aligned}
\mu= & 3 g \varepsilon_{\mathrm{dd}} \frac{n_{0} \kappa_{x} \kappa_{y}}{2 R_{z}^{2}}\left[R_{z}^{2} \beta_{001}-\beta_{101} x^{2}-\beta_{011} y^{2}-3 \beta_{002} z^{2}\right] \\
& +V(\mathbf{r})+\left(1-\varepsilon_{\mathrm{dd}}\right) \frac{g n_{0}}{R_{z}^{2}}\left(R_{z}^{2}-\frac{x^{2}}{\kappa_{x}^{2}}-\frac{y^{2}}{\kappa_{y}^{2}}-z^{2}\right) .
\end{aligned}
$$

Inspection of the coefficients of $x^{2}, y^{2}$, and $z^{2}$ leads to three self-consistency relations, given by

$$
\begin{gathered}
\kappa_{x}^{2}=\frac{\omega_{z}^{2}}{\omega_{x}^{2}} \frac{1+\varepsilon_{\mathrm{dd}}\left(\frac{3}{2} \kappa_{x}^{3} \kappa_{y} \beta_{101}-1\right)}{1-\varepsilon_{\mathrm{dd}}\left(1-\frac{9 \kappa_{x} \kappa_{y}}{2} \beta_{002}\right)}, \\
\kappa_{y}^{2}=\frac{\omega_{z}^{2}}{\omega_{y}^{2}} \frac{1+\varepsilon_{\mathrm{dd}}\left(\frac{3}{2} \kappa_{y}^{3} \kappa_{x} \beta_{011}-1\right)}{1-\varepsilon_{\mathrm{dd}}\left(1-\frac{9 \kappa_{x} \kappa_{y}}{2} \beta_{002}\right)}, \\
R_{z}^{2}=\frac{2 g n_{0}}{m \omega_{z}^{2}}\left[1-\varepsilon_{\mathrm{dd}}\left(1-\frac{9 \kappa_{x} \kappa_{y}}{2} \beta_{002}\right)\right] .
\end{gathered}
$$

Solving Eqs. (15)-(17) gives the exact static solutions of the system in the TF regime.

The energetic stability of the condensate is determined by the TF energy functional

$$
E=\int\left[V(\mathbf{r})+\frac{1}{2} \Phi_{\mathrm{dd}}(\mathbf{r})+\frac{1}{2} g n(\mathbf{r})\right] n(\mathbf{r}) d^{3} r .
$$

Inserting the parabolic density profile (10) yields an energy landscape

$$
\begin{aligned}
E= & \frac{15 N^{2} g}{28 \pi \kappa_{x} \kappa_{y} R_{z}^{3}}\left[\left(1-\varepsilon_{\mathrm{dd}}\right)+\frac{3}{8} \kappa_{x} \kappa_{y} \varepsilon_{\mathrm{dd}}\left(7 \beta_{001}-3 \beta_{002}\right.\right. \\
& \left.\left.-\kappa_{x}^{2} \beta_{101}-\kappa_{y}^{2} \beta_{011}\right)\right]+\frac{N}{14} m R_{z}^{2}\left(\kappa_{x}^{2} \omega_{x}^{2}+\kappa_{y}^{2} \omega_{y}^{2}+\gamma^{2}\right) .
\end{aligned}
$$

Static solutions correspond to stationary points in the energy landscape. If the stationary point is a local minimum in the energy landscape, it corresponds to a physically stable solution. However, if the stationary point is a maximum or a saddle point, the corresponding solution will be energetically unstable. The nature of the stationary point can be determined by performing a second derivative test on Eq. (19) with respect to the variables $\kappa_{x}, \kappa_{y}$, and $R_{z}$. This leads to six lengthy equations that will not be presented here. Note that this only determines whether the stationary point is a local minimum within the class of parabolic density profiles. In other words, with the three variables $\kappa_{x}, \kappa_{y}$, and $R_{z}$ we are only able to determine stability against "scaling" fluctuations, so named because they correspond to a rescaling of the static solution $[63,64]$. However, the class of scaling fluctuations includes important low-lying shape oscillations such as the monopole and quadrupole modes. Although higher order (beyond quadrupole) modes can become unstable in certain regimes, as a criterion of stability we will use the local minima of (19). This assumption is supported by the recent experiments by Koch et al. [11], where dipolar BECs were produced with $\varepsilon_{\mathrm{dd}}>1$ that were stable over significant time scales.

\section{B. Cylindrically symmetric static solutions for $g>0$, and the critical trap ratios $\gamma_{\text {crit }}^{+}$and $\gamma_{\text {crit }}^{-}$}

We have obtained the static solutions for a cylindrically symmetric BEC by solving Eqs. (15)-(17) numerically. The solutions behave differently depending on whether the $s$ wave interactions are repulsive or attractive. We begin by considering the $g>0$ case. The ensuing static solutions, characterized by their aspect ratio $\kappa$, are presented in Fig. 2 as a function of $\varepsilon_{\mathrm{dd}}$ with each line representing a different trap ratio $\gamma$. While the TF solutions in the regime $\varepsilon_{\mathrm{dd}}>0$ have been discussed previously [26,27], the regime of $\varepsilon_{\mathrm{dd}}<0$ has not been studied. Be aware that when we fix $g>0$, the regime $\varepsilon_{\mathrm{dd}}<0$ (left-hand side of Fig. 2) corresponds to $C_{\mathrm{dd}}<0$ where the dipolar interaction is reversed, repelling along $z$ and attracting in the transverse direction. This can be achieved by rapid rotation of the field aligning the dipoles about the $z$ axis [16].

Before we examine the question of stability, let us first interpret the structure of the solutions shown in Fig. 2. Imagine an experiment in which the magnitude of $\varepsilon_{\mathrm{dd}}$ is 


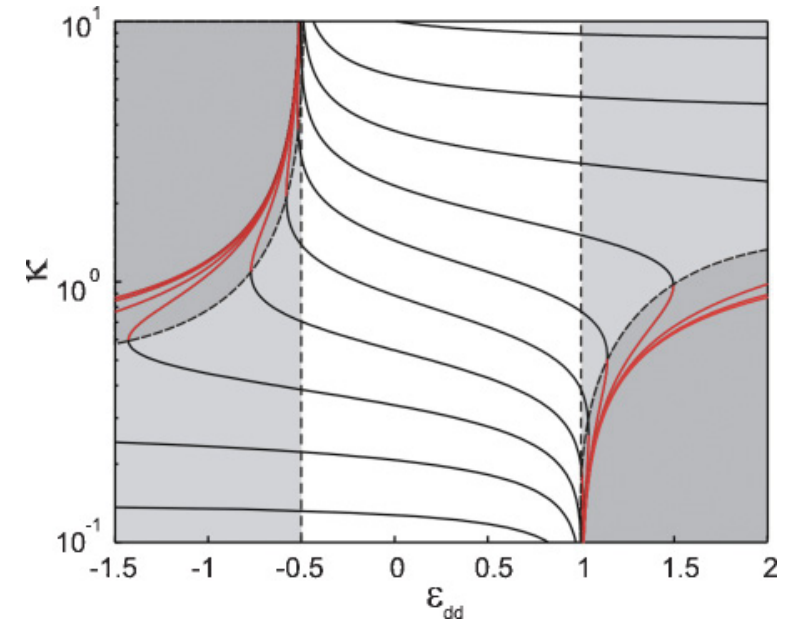

FIG. 2. (Color online) Aspect ratio $\kappa$ of the $g>0$ cylindrically symmetric static solutions as a function of $\varepsilon_{\mathrm{dd}}$ according to Eqs. (15)(17). Note that $\varepsilon_{\mathrm{dd}}<0$ corresponds to $C_{\mathrm{dd}}<0$. The solid lines indicate the static solutions for specific trap ratios $\gamma$ which are equally spaced on a logarithmic scale in the range $\gamma=[0.1,10]$. The parameter space of global, metastable, and unstable solutions is denoted by white, light gray, and dark gray regions, respectively.

slowly increased from zero. At $\varepsilon_{\mathrm{dd}}=0$ we have purely $s$-wave interactions, and all solutions have the same aspect ratio as the trap, i.e. $\kappa=\gamma$. As $\varepsilon_{\mathrm{dd}}$ is increased above zero, $\kappa$ decreases so that $\kappa<\gamma$ for all solutions. This is because standard magnetostriction causes dipolar BECs to be more cigar shaped than their $s$-wave counterparts. Conversely, if $\varepsilon_{\mathrm{dd}}$ is made negative, then $\kappa$ increases so that $\kappa>\gamma$ for all solutions. This is because when $C_{\mathrm{dd}}<0$ we have nonstandard (reversed) magnetostriction which leads to a more pancake-shaped BEC.

Consider now the stability of the solutions, beginning with the range $-1 / 2<\varepsilon_{\mathrm{dd}}<1$ (white region in Fig. 2). We find that the energy landscape (19) has only one stationary point, namely, a global energy minimum, and it occurs at finite values of the radii $R_{x}\left(=R_{y}\right)$ and $R_{z}$. This global minimum persists for all trap ratios (outside of the range $-1 / 2<\varepsilon_{\mathrm{dd}}<1$ the existence of stable static solutions depends on $\gamma$ ). Thus, in the range $-1 / 2<\varepsilon_{\mathrm{dd}}<1$ the static TF solution is stable against scaling fluctuations. Other classes of perturbation could lead to instability, but there is good reason to believe that in this range the parabolic solution is also stable against these. Take, for example, phonons, i.e., local density perturbations. These have a character that can be considered opposite to the global motion involved in scaling oscillations. The local character of phonons means that considerable insight can be gained from the limiting case of a homogeneous dipolar condensate. The energy of a plane wave perturbation (phonon) with momentum $p$ is given by the Bogoliubov energy $E_{\mathrm{B}}[12]$,

$$
E_{\mathrm{B}}^{2}=\left(\frac{p^{2}}{2 m}\right)^{2}+2 g n\left[1+\varepsilon_{\mathrm{dd}}\left(3 \cos ^{2} \theta-1\right)\right] \frac{p^{2}}{2 m},
$$

where $\theta$ is the angle between the momentum of the phonon and the polarization direction. The perturbation evolves as $\sim \exp \left(-i E_{\mathrm{B}} t / \hbar\right)$, and so when $E_{\mathrm{B}}^{2}<0$ the perturbations grow exponentially, signifying a dynamical instability. Dynamical stability requires that $E_{\mathrm{B}}^{2}>0$ which, for $g>0$, corresponds to the requirement that $\left[1+\varepsilon_{\mathrm{dd}}\left(3 \cos ^{2} \theta-1\right)\right] \geqslant 0$ in Eq. (20). This leads once again to precisely the stability condition $-1 / 2<\varepsilon_{\mathrm{dd}}<1$.

Outside of the regime $-1 / 2<\varepsilon_{\mathrm{dd}}<1$ the global energy minimum of the TF system is a collapsed state where at least one of the radii is zero, just like in the uniform dipolar BEC case. However, unlike the uniform case, in the presence of a trap the energy functional can also support a local energy minimum corresponding to a metastable solution (light gray region in Fig. 2). The existence of a metastable solution means there must also be a saddle point connecting the metastable solution to the collapsed state, and this is indicated by the dark gray region in Fig. 2.

In general, the occurrence of metastable solutions depends sensitively on $\varepsilon_{\mathrm{dd}}$ and $\gamma$. Remarkably, however, there are two critical trap ratios, $\gamma_{\text {crit }}^{+}=5.17$ and $\gamma_{\text {crit }}^{-}=0.19$, beyond which the BEC is stable against scaling fluctuations even as the strength of the dipolar interactions becomes infinite. First consider $\varepsilon_{\mathrm{dd}}>1$, for which there is a susceptibility for collapse toward an infinitely narrow line of end-to-end dipoles $\left(R_{x}=R_{y} \rightarrow 0\right)$. Providing $\gamma>\gamma_{\text {crit }}^{+}$, i.e., if the trap is pancake enough, condensate solutions metastable against scaling fluctuations persist even as $\varepsilon_{\mathrm{dd}} \rightarrow \infty$ [13,14,27]. Referring to Fig. 2, these curves are located in the upper right-hand portion of the plot and asymptote to horizontal lines as $\varepsilon_{\mathrm{dd}}$ is increased (see Fig. 3 in [27] for a plot which extends $\varepsilon_{\mathrm{dd}}$ to much higher values than shown here so that this behavior is clearer). However, if the trap is not pancake-shaped enough, i.e., $\gamma<\gamma_{\text {crit }}^{+}$, then as $\varepsilon_{\mathrm{dd}}$ is increased from zero the local energy minimum eventually disappears and no stable solutions exist. Referring again to Fig. 2, these are the curves that turn over as $\varepsilon_{\mathrm{dd}}$ is increased, and in so doing enter the dark gray region. Second, consider $\varepsilon_{\mathrm{dd}}<-0.5$, for which the system is susceptible to collapse into an infinitely thin pancake of side-by-side dipoles $\left(R_{z} \rightarrow 0\right)$. If the trap is sufficiently cigarlike with $\gamma<\gamma_{\text {crit }}^{-}$, then collapse via scaling oscillations is suppressed even in the limit $\varepsilon_{\mathrm{dd}} \rightarrow-\infty$. These curves are located in the lower left-hand portion of Fig. 2 and asymptote to horizontal lines. However, if the trap is not cigar-shaped enough, i.e., $\gamma>\gamma_{\text {crit }}^{-}$, then for sufficiently large and negative $\varepsilon_{\mathrm{dd}}$ the metastable solution disappears, bending upward to enter the dark gray region on the left-hand portion of Fig. 2 and the system becomes unstable to collapse.

In a recent experiment Lahaye et al. [10] measured the aspect ratio of the dipolar condensate over the range $0 \lesssim \varepsilon_{\mathrm{dd}} \lesssim$ 1 , using a Feshbach resonance to tune $g$, and found very good agreement with the TF predictions. Similarly, Koch et al. [11] observed the threshold for collapse in a $\gamma=1$ system to be $\varepsilon_{\mathrm{dd}} \approx 1.1$, in excellent agreement with the TF prediction of $\varepsilon_{\mathrm{dd}}=1.06$. Using various trap ratios, it was also found that collapse became suppressed in flattened geometries, and the critical trap ratio was observed to exist in the range $\gamma_{\text {crit }}^{+} \approx 5-$ 10 , which is in qualitative agreement with the TF predictions.

\section{Cylindrically symmetric static solutions for $\boldsymbol{g}<\mathbf{0}$, and the nature of dipolar stabilization}

We now consider the case of attractive $s$-wave interactions $g<0$. Negative values of $g$ can be achieved using a Feshbach resonance. The static solutions are presented in Fig. 3. Be 


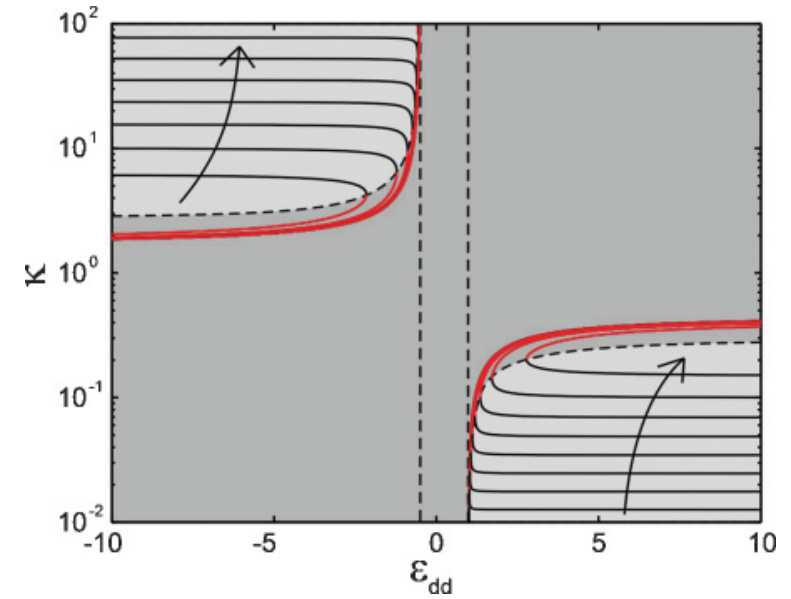

FIG. 3. (Color online) Aspect ratio $\kappa$ of the $g<0$ cylindrically symmetric static solutions as a function of $\varepsilon_{\mathrm{dd}}$. Note that the regime of $\varepsilon_{\mathrm{dd}}>0$ corresponds to $C_{\mathrm{dd}}<0$. The solid lines denote static solutions for specific trap ratios $\gamma$, equally spaced on a logarithmic scale in the ranges $\gamma=\left[0.010, \gamma_{\text {crit }}^{-}\right]$(lower right set of curves) and $\gamma=\left[\gamma_{\text {crit }}^{+}, 100\right]$ (upper left set of curves). Arrows indicate direction of increasing $\gamma$. The black lines in the light gray regions correspond to local minima (metastable) points in the energy landscape, while the red lines in the dark gray region correspond to saddle (unstable) points. At the extreme left- and right-hand sides of the figure the stable solutions become horizontal lines as they tend asymptotically to the trap aspect ratio $\kappa \rightarrow \gamma$ (see text).

aware that because $g<0, \varepsilon_{\mathrm{dd}}<0\left(\varepsilon_{\mathrm{dd}}>0\right)$ now corresponds to $C_{\mathrm{dd}}>0\left(C_{\mathrm{dd}}<0\right)$. The stability diagram differs greatly from the $g>0$ case and, in particular, no TF solutions exist in the range $-1 / 2<\varepsilon_{\mathrm{dd}}<1$. Nevertheless, TF solutions can exist outside of this range in regions of parameter space determined by the two critical trap ratios $\gamma_{\text {crit }}^{-}$and $\gamma_{\text {crit }}^{+}$ introduced in the previous section. We find that for $\varepsilon_{\mathrm{dd}}>0$, solutions only exist for significantly cigar-shaped geometries with $\gamma<\gamma_{\text {crit }}^{-}=0.19$; while for $\varepsilon_{\mathrm{dd}}<0$, solutions only exist for significantly pancake-shaped geometries with $\gamma>\gamma_{\text {crit }}^{+}=$ 5.17. Furthermore, the attractive $s$-wave interactions always cause the global minimum to be a collapsed state. This means that static TF solutions are only ever metastable (light gray region in Fig. 3) when $g<0$.

Although our TF model predicts that no solutions exist for $-1 / 2<\varepsilon_{\mathrm{dd}}<1$, it is well known that stable condensates with attractive purely $s$-wave interactions (corresponding to $\left.\varepsilon_{\mathrm{dd}}=0\right)$ can be made in the laboratory [65]. The zero-point energy of the atoms (ignored in the TF model) induced by the trapping potential stabilizes the condensate up to a critical number of atoms $N_{\text {crit }}=k a_{\mathrm{HO}} /\left|a_{s}\right|$, where $a_{\mathrm{HO}}=\sqrt{\hbar /(m \bar{\omega})}$ is the harmonic oscillator length obtained from the mean trapping frequency $\bar{\omega}=\left(\omega_{x} \omega_{y} \omega_{z}\right)^{1 / 3}$, and $k \approx 1 / 2$ is a constant [66]. One can expect, therefore, that for a finite number of atoms the presence of zero-point energy enhances the stability of the condensate beyond the TF solutions [32]. This will extend somewhat the light gray regions in both Figs. 2 and 3 into the dark gray regions, the amount depending upon $N_{\text {crit }} / N$. The TF regime corresponds to the $N \rightarrow \infty$ limit and so is in a sense a universal regime that can always be realized with a large enough condensate.
Insight into the TF stability diagram shown in Fig. 3 can once again be gleaned from the Bogoliubov spectrum for a uniform system given by Eq. (20), this time with $g<0$. First, for the purely $s$-wave case we recall the well-known result [3] that a homogeneous attractive BEC is always unstable to collapse. With dipolar interactions the uniform system is stable to axial perturbations $(\theta=0)$ for $\varepsilon_{\mathrm{dd}}<-1 / 2$ and to radial perturbations $(\theta=\pi / 2)$ for $\varepsilon_{\mathrm{dd}}>1$. This is the exact opposite of the $g>0$ case and corroborates the lack of solutions given by the TF equations for $-1 / 2<\varepsilon_{\mathrm{dd}}<1$. Of course, $\varepsilon_{\mathrm{dd}}<-1 / 2$ and $\varepsilon_{\mathrm{dd}}>1$ cannot be simultaneously satisfied, and so a uniform dipolar system with $g<0$ is always unstable. However, when the system is trapped the condensate can be stabilized even in the TF regime. The mean dipolar interaction depends on the condensate shape and can become net repulsive in cigar-shaped systems when $\varepsilon_{\mathrm{dd}}>0$ (for which $C_{\mathrm{dd}}<0$ ), and in pancake-shaped systems when $\varepsilon_{\mathrm{dd}}<0$ (for which $C_{\mathrm{dd}}>0$ ). Remarkably, in these cases it is the dipolar interactions that stabilize the BEC against the attractive $s$-wave interactions and lead to the regions of metastable static solutions observed in Fig. 3. Without the dipolar interactions the BEC would collapse.

The metastable TF solutions shown in Fig. 3 have a counter-intuitive dependence upon $\varepsilon_{\mathrm{dd}}$. Take, for example, the family of metastable solutions (black curves) in the lower right-hand portion of the figure. We see that as $\varepsilon_{\mathrm{dd}}$ increases, $\kappa$ decreases (condensate becomes more cigar shaped). This is in contradiction to what one might naively expect, because on this side of the figure $C_{\mathrm{dd}}<0$, and so the dipolar interaction has an energetic preference for dipoles sitting side-by-side not end-to-end! In order to appreciate what is happening in this region of Fig. 3, observe that for each value of $\varepsilon_{\mathrm{dd}}$ there is a critical value of the condensate aspect ratio $\kappa$ below which the system is metastable, and above which it is unstable. As $\varepsilon_{\mathrm{dd}}$ is increased from this point the net repulsive dipolar interactions favor elongating the BEC so that atoms sit farther from each other, thereby lowering the interaction energy and decreasing $\kappa$. In the limit $\kappa \rightarrow 0$, one can show that the dipolar mean-field potential tends to $\Phi_{\mathrm{dd}}=-g \varepsilon_{\mathrm{dd}} n(\mathbf{r})$ [34]; i.e., it behaves like a spherically symmetric contact interaction which is repulsive when $g<0$ and $\varepsilon_{\mathrm{dd}}>0$. This means that when $\varepsilon_{\mathrm{dd}}$ is increased in a strongly cigar-shaped configuration the condensate aspect ratio tends asymptotically toward that of the trap $\kappa \rightarrow \gamma$, as it must for a system with net-repulsive spherically symmetric contact interactions. This behavior can be seen in Fig. 3 where the black curves all tend to straight lines as $\varepsilon_{\mathrm{dd}}$ is increased, and the asymptotic value of $\kappa$ they tend to is exactly the trap aspect ratio $\gamma$.

A parallel argument holds for the upper left-hand portion of Fig. 3 where the condensate is quite strongly pancake shaped $\left(\gamma>\gamma_{\text {crit }}^{+}\right)$: in the limit $\kappa \rightarrow \infty$, one can show that the dipolar mean-field potential tends to $\Phi_{\mathrm{dd}}=2 g \varepsilon_{\mathrm{dd}} n(\mathbf{r})$ [34]; i.e., it behaves like a spherically symmetric contact interaction which is repulsive when $g<0$ and $\varepsilon_{\mathrm{dd}}<0$. In this portion of the figure, one therefore also finds that as $\left|\varepsilon_{\mathrm{dd}}\right| \rightarrow \infty$ the condensate aspect ratio tends asymptotically toward that of the $\operatorname{trap} \kappa \rightarrow \gamma$.

It is tempting to conclude that when $g<0$ the collapse that occurs as the strength of the dipolar interactions is reduced relative to the $s$-wave interactions is an " $s$-wave collapse" of 
the type encountered in BECs with attractive purely $s$-wave interactions [65], which typically occurs through an unstable monopole mode [67]. However, from Fig. 3 we see that the magnitude of the dipolar interaction is always finite at the collapse point. Furthermore, we shall find in subsequent sections that it is always a quadrupole mode that is responsible for collapse in a TF dipolar BEC. Collapse via a quadrupole mode has a one- or two-dimensional character, depending on the sign of $C_{\mathrm{dd}}$ [32], and is distinct from collapse via the monopole mode which has a three-dimensional character.

In the experiment by Koch et al. [11], where the critical scattering length $a_{\text {crit }}$ at which collapse occurs in a dipolar BEC was measured for different trapping ratios $\gamma$, there are a few data points corresponding to negative values of $g$ for strongly oblate condensates, and are thus of relevance to this section. We infer from their Fig. 3 that collapse occurred when $\varepsilon_{\mathrm{dd}} \gtrsim-7$ in a trap with $\gamma=10$. However, for this trap the TF static solutions only disappear at $\varepsilon_{\mathrm{dd}} \gtrsim-1.5$ and the inclusion of zero-point motion cannot explain this discrepancy between theory and experiment since it should increase the critical value of $\varepsilon_{\mathrm{dd}}$ above -1.5 , not decrease it. Furthermore, including the zero-point motion by using a Gaussian ansatz leads to an almost identical theoretical prediction [11]. Possible explanations for the discrepancy include (i) the errors bars on their data imply that $-5 \gtrsim a_{\text {crit }} / a_{0} \gtrsim 1$ which, due to the inverse relation between $\varepsilon_{\mathrm{dd}}$ and $g$, leads to a huge uncertainty in $\varepsilon_{\mathrm{dd}}$; (ii) they report an uncertainty in their trapping frequencies of $0.94<\omega_{x} / \omega_{y}<1.04$, which translates into an uncertainty in the ellipticity of the trap in the $x-y$ plane of $-0.04<\epsilon<0.06$ (as we shall see in Sec. IID below, this can have an effect upon the stability); and (iii) very close to collapse the dominant dipolar interactions may lead to significant deviations of the density profile from a single-peaked inverted parabola or Gaussian profile; for example, Ronen et al. [29] have predicted biconcave density structures. These may alter the stability properties of the condensate.

Having indicated how the static solutions behave for attractive $s$-wave interactions $g<0$, for the remainder of the paper we will concentrate (although not exclusively) on the more common case of repulsive $s$-wave interactions.

\section{Non-cylindrically-symmetric static solutions}

We now consider the more general case of a noncylindrically-symmetric system for which the trap ellipticity $\epsilon$ is finite and $\kappa_{x}$ and $\kappa_{y}$ typically differ. Note that we perform our analysis of non-cylindrically-symmetric static solutions for repulsive $s$-wave interactions $g>0$. In Fig. 4 we show how $\kappa_{x}$ and $\kappa_{y}$ vary as a function of $\varepsilon_{\mathrm{dd}}$ in a non-cylindricallysymmetric trap. Different values of trap ratio are considered and generic qualitative features exist. The splitting of $\kappa_{x}$ and $\kappa_{y}$ is evident, with $\kappa_{x}$ shifting upward and $\kappa_{y}$ shifting downward in comparison to the cylindrically symmetric solutions. Furthermore, the branches become less stable to collapse. For example, for $\gamma=0.18<\gamma_{\text {crit }}^{-}$[Fig. 4(a)], in the cylindrically symmetric system there exist stable solutions for $\varepsilon_{\mathrm{dd}} \rightarrow-\infty$, but in the anisotropic case, stationary solutions only exist up to $\varepsilon_{\mathrm{dd}} \simeq-11$.

We already noted in the Introduction that for a cylindrically symmetric dipolar BEC, magnetostriction causes the radial
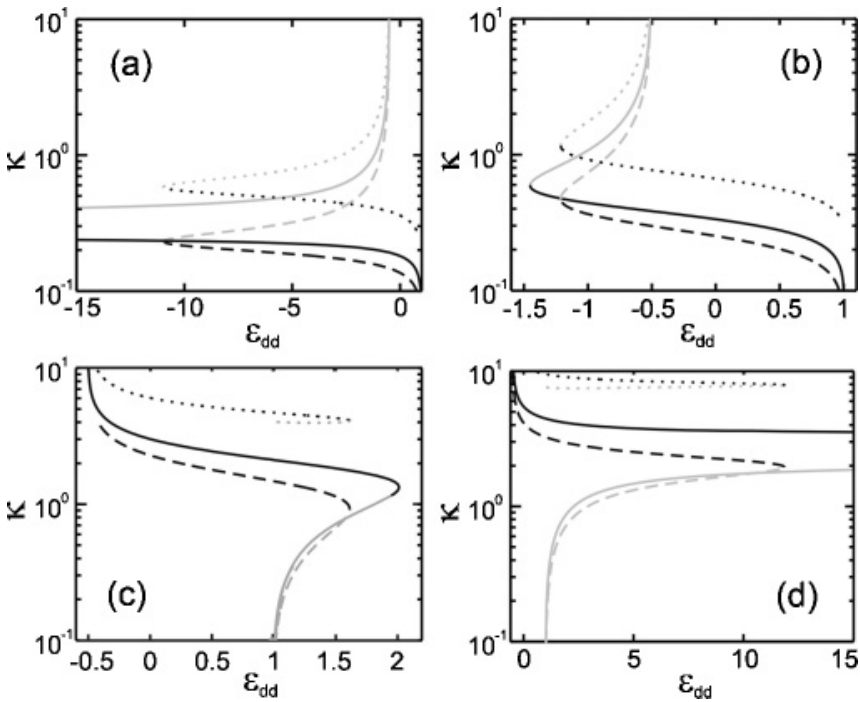

FIG. 4. Stable static solutions, characterized by the aspect ratios $\kappa_{x}$ (dotted lines) and $\kappa_{y}$ (dashed lines), in a non-cylindricallysymmetric trap with ellipticity $\epsilon=0.75$ and (a) $\gamma=0.18$, (b) $\gamma=$ 0.333 , (c) $\gamma=3$, and (d) $\gamma=5.5$. Stable (unstable) static solutions are indicated by black (gray) lines. The corresponding static solutions for $\epsilon=0$ are indicated by solid lines.

versus axial aspect ratio $\kappa=R_{x} / R_{z}$ to differ from the trap ratio $\gamma$, in contrast to a pure $s$-wave BEC for which $\kappa=\gamma$. It is therefore interesting to note that we find that when the trap is not cylindrically symmetric, a dipolar BEC also has an ellipticity in the $x-y$ plane which differs from that of the trap, although the deviation is generally small. This occurs despite the fact that dipolar interactions are radially symmetric.

\section{CALCULATION OF THE EXCITATION SPECTRUM}

Now that we have exhibited some of the features of the static solutions in the TF regime, we wish to determine their excitation spectrum. The methods which have been previously used for finding the excitation spectrum of a dipolar BEC include (i) a variational approach applied to a Gaussian approximation for the BEC density profile $[14,25,48,68]$, which allows one to derive equations of motion for the widths of the Gaussian; (ii) using the equations of dissipationless hydrodynamics, namely, the continuity and Euler equations, to obtain equations of motion for the TF radii [26,51]; this method is exact in the TF limit (recall that the TF regime is mathematically identical to the hydrodynamics of superfluids at zero temperature); (iii) solving the full Bogoliubov equations [28,49,50]; and (iv) solving for the time evolution of the full time-dependent GPE under well-chosen perturbations $[14,25,48]$.

Methods (i) and (ii) are simple but yield only the three lowest energy collective modes (the monopole and two quadrupole modes). However, in the pure $s$-wave case these methods do have the advantage of giving analytic expressions for the frequencies, and in the dipolar case the frequencies are given by the solution of the algebraic equations (15)-(17), which are simple to solve. This is to be contrasted with the other methods which, although more general, require much more sophisticated numerical approaches. Furthermore, the nonlocal nature of the dipolar interactions make numerical 
calculations considerably more intensive than their $s$-wave equivalents. Therefore, the approach we adopt here is semianalytic, incorporating analytic results for the nonlocal dipolar potential, thereby reducing the problem to the solution of (local) algebraic equations.

In our approach we generalize the methodology previously applied by Sinha and Castin [42] to pure $s$-wave BECs, where linearized equations of motion are derived for small perturbations about the mean-field stationary solution. One strength of this method, in contrast to some of those mentioned above, is that it is trivially extended to arbitrary modes of excitation and unstable modes or dynamical instability. For example, extension of the variational approach to higher-order modes (e.g., to consider the scissors modes of an $s$-wave BEC [69]) requires that this is "built in" to the variational ansatz itself. We outline our approach below.

The dynamics of the condensate wave function $\psi(\mathbf{r}, t)$ is described by the time-dependent Gross-Pitaevskii equation

$$
i \hbar \frac{\partial \psi}{\partial t}=\left(-\frac{\hbar^{2}}{2 m} \nabla^{2}+V+\Phi_{\mathrm{dd}}+g|\psi|^{2}\right) \psi,
$$

where, for convenience, we have dropped the arguments $\mathbf{r}$ and $t$. By expressing $\psi$ in terms of its density $n$ and phase $S$ as

$$
\psi=\sqrt{n} e^{i S},
$$

one obtains from Eq. (21) the well-known hydrodynamic equations

$$
\begin{gathered}
\frac{\partial n}{\partial t}=-\frac{\hbar}{m} \nabla \cdot(n \nabla S), \\
\hbar \frac{\partial S}{\partial t}=-\frac{\hbar^{2}}{2 m}|\nabla S|^{2}-V-g n-\Phi_{\mathrm{dd}} .
\end{gathered}
$$

We have dropped the term $\left(\hbar^{2} / 2 m \sqrt{n}\right) \nabla^{2} \sqrt{n}$ arising from density gradients - this is synonymous with making the TF approximation [3]. Note that static solutions satisfy the equilibrium conditions $\partial n / \partial t=0$ and $\partial S / \partial t=-\mu / \hbar$.

We now consider small perturbations of the density $\delta n$ and phase $\delta S$ about the TF static solutions found in Sec. II. The static solutions for a nonrotating condensate have $S=0$ and $n=n_{\text {eq }}$, where $n_{\text {eq }}$ is the parabolic density profile given in Eq. (10) and is obtained by solving Eqs. (15)-(17). Linearizing the hydrodynamic equations (22) and (23) in the perturbations, we find that the dynamics are governed by

$$
\frac{\partial}{\partial t}\left[\begin{array}{l}
\delta S \\
\delta n
\end{array}\right]=\mathcal{L}\left[\begin{array}{l}
\delta S \\
\delta n
\end{array}\right]
$$

where

$$
\mathcal{L}=-\left[\begin{array}{cc}
0 & g\left(1+\varepsilon_{\mathrm{dd}} K\right) / \hbar \\
\frac{\hbar}{m} \nabla \cdot n_{\mathrm{eq}} \nabla & 0
\end{array}\right] .
$$

Note that some of the constants appearing in Eq. (25) were misquoted in our previous papers, although the results given in those papers are not affected [70]. The operator $K$ in Eq. (25) is defined via its action upon $\delta n$ as

$$
(K \delta n)(\mathbf{r})=-3 \frac{\partial^{2}}{\partial z^{2}} \int \frac{\delta n\left(\mathbf{r}^{\prime}\right) d^{3} r^{\prime}}{4 \pi\left|\mathbf{r}-\mathbf{r}^{\prime}\right|}-\delta n(\mathbf{r}) .
$$

The integral in the above expression is carried out over the domain where the unperturbed density given by Eq. (10) satisfies $n_{\text {eq }}>0$, that is, the general ellipsoidal domain with radii $R_{x}, R_{y}, R_{z}$. Extending the integration domain to the region where $n_{\text {eq }}+\delta n>0$ would only add $O\left(\delta n^{2}\right)$ effects, since it is exactly in this extended domain that $n=O(\delta n)$, whereas the size of the extension is also proportional to $\delta n$. Clearly, to first order in $\delta n$, the quantity $\varepsilon_{\mathrm{dd}} K \delta n$ is the dipolar potential associated with the density distribution $\delta n$. To obtain the global shape excitations of the BEC, one has to find the eigenfunctions $\delta n$ and $\delta S$ and eigenvalues $\lambda$ of operator $\mathcal{L}$ of Eq. (25). For such eigenfunctions, Eq. (24) trivially yields an exponential time evolution of the form $\sim \exp (\lambda t)$. When the associated eigenvalue $\lambda$ is imaginary, the eigenfunction corresponds to a time-dependent oscillation of the BEC. However, when $\lambda$ possesses a positive real part, the eigenfunction represents an unstable excitation which grows exponentially. Such dynamical instabilities are an important consideration, for example, in rotating condensates where they initiate vortex lattice formation $[42,43]$. However, in the current study we will focus on stable excitations of nonrotating systems.

To find such eigenfunctions and eigenvalues we consider a polynomial ansatz for the perturbations in the coordinates $x, y$, and $z$, of a total degree $v$ [42], that is,

$$
\delta n=\sum_{p, q, r} a_{p q r} x^{p} y^{q} z^{r}, \quad \delta S=\sum_{p, q, r} b_{p q r} x^{p} y^{q} z^{r},
$$

where

$$
v=\max _{\substack{a_{p q r} \neq 0 \\ b_{p q r} \neq 0}}\{p+q+r\} .
$$

All operators in Eq. (25), acting on such polynomials of degree $v$, result again in polynomials of the same order. For the operator $K$ this property might not be obvious, but a remarkable result known from 19th-century gravitational potential theory states that the integral in Eq. (26) evaluated for a polynomial density $\delta n$, yields another polynomial in $x, y$, and $z$. Its coefficients are given in terms of the integrals $\beta_{i j k}$ defined in Eq. (12), and the exact expressions are presented in Appendix B. The degree of the resulting polynomial is $v+2$, and taking the derivative with respect to $z$ twice yields another polynomial of degree $v$ again. Thus, operator (25) can be rewritten as a matrix mapping between scalar vectors of polynomial coefficients. Numerically finding the eigenvalues and eigenvectors of such a system is a simple task, which computational packages can typically perform.

We present only the lowest lying shape oscillations corresponding to polynomial phase and density perturbations of degrees $v=1$ and $v=2$. These form the monopole, dipole, quadrupole, and scissors modes. These excitations are illustrated schematically in Fig. 1 and described below, where we state only the form of the density perturbation $\delta n$, since it can be shown that the corresponding phase perturbation $\delta S$ always contains the same monomial terms. Note that $a, b, c$ and $d$ are positive real coefficients.

(1) Dipole modes $D_{x}, D_{y}$, and $D_{z}$ : A center-of-mass motion along each trap axis [71]. The $D_{x}$ mode, for instance, is characterized by $\delta n= \pm a x$.

(2) Monopole mode $M:$ An in-phase oscillation of all radii with the form $\delta n= \pm a \pm\left(b x^{2}+c y^{2}+d z^{2}\right)$. 
(3) Quadrupole modes $Q_{1}^{x y}, Q_{1}^{x z}$, and $Q_{1}^{y z}$ : The $Q_{1}$ modes feature two radii oscillating in phase with each other (denoted in superscripts) and out of phase with the remaining radius. For example, the $Q_{1}^{x y}$ mode is characterized by $\delta n= \pm a \pm$ $\left(b x^{2}+c y^{2}-d z^{2}\right)$.

(4) Quadrupole mode $Q_{2}$ : This two-dimensional mode is supported only in a plane where the trapping has circular symmetry. For example, in the transverse plane of a cylindrically symmetric system the transverse radii oscillate out of phase with each other, with no motion in $z$, according to $\delta n= \pm a(x \pm i y)^{2}$.

(5) Scissors modes $\mathrm{Sc}_{x y}, \mathrm{Sc}_{y z}$, and $\mathrm{Sc}_{x z}$ : Shape-preserving oscillatory rotation of the BEC over a small angle in the $x y, x z$, and $y z$ plane, respectively. The $\mathrm{Sc}_{x y}$ mode is characterized by $\delta n= \pm a x y$. Note that a scissors mode in a given plane requires that the condensate asymmetry in that plane is nonzero, otherwise no cross terms exist. Furthermore, the amplitude of the cross terms should remain smaller than the condensate or trap asymmetry, otherwise the scissors mode turns into a quadrupole mode [38].

Note that in order to confirm the dynamical stability of the solution, one must also check that positive eigenvalues do not exist. We have performed this throughout this paper and consistently observe that when $\operatorname{Im}(\lambda) \neq 0$ then $\operatorname{Re}(\lambda)=0$, and when $\operatorname{Im}(\lambda)=0, \operatorname{Re}(\lambda) \neq 0$. It is also possible to determine excitation frequencies of higher-order excitations of the BEC by including higher-order monomial terms. Such modes, for example, play an important role in the dynamical instability of rotating systems [35,42].

We would like to remind the reader that they can download the MATLAB program [52] used to perform the calculations described in this section. It includes an easy-to-use graphical user interface.

\section{EXCITATIONS IN A CYLINDRICALLY SYMMETRIC TRAP}

In this section we present the oscillation frequencies of the lowest lying stable excitations of a dipolar condensate in a cylindrically symmetric trap. Through specific examples we indicate how they behave with the key experimental parameters, namely, the dipolar interaction strength $\varepsilon_{\mathrm{dd}}$ and trap ratio $\gamma$. Note that we will discuss the scissors modes in more detail in Sec. VI. Here we will just point out that two scissors modes exist, corresponding to $\mathrm{Sc}_{x z}$ and $\mathrm{Sc}_{y z}$, while the $\mathrm{Sc}_{x y}$ mode is nonexistent due to the cylindrical symmetry of the system.

\section{A. Variation with dipolar interactions $\varepsilon_{\mathrm{dd}}$ for $\boldsymbol{g}>\mathbf{0}$}

In Fig. 5 we show how the collective-mode frequencies vary with the dipolar interactions for the case of $g>0$. Although it would seem experimentally relevant to present these frequencies as a function of $\varepsilon_{\mathrm{dd}}$, we plot them as a function of the aspect ratio $\kappa$ instead. We do this for the following two reasons: (i) plotting the frequencies as a function of $\varepsilon_{\mathrm{dd}}$ is problematic since two static solutions (metastable local minima and unstable saddle points) can exist for a given value of $\varepsilon_{\mathrm{dd}}$, and (ii) in the critical region of collapse at the turning point from stable to unstable, the excitation frequencies
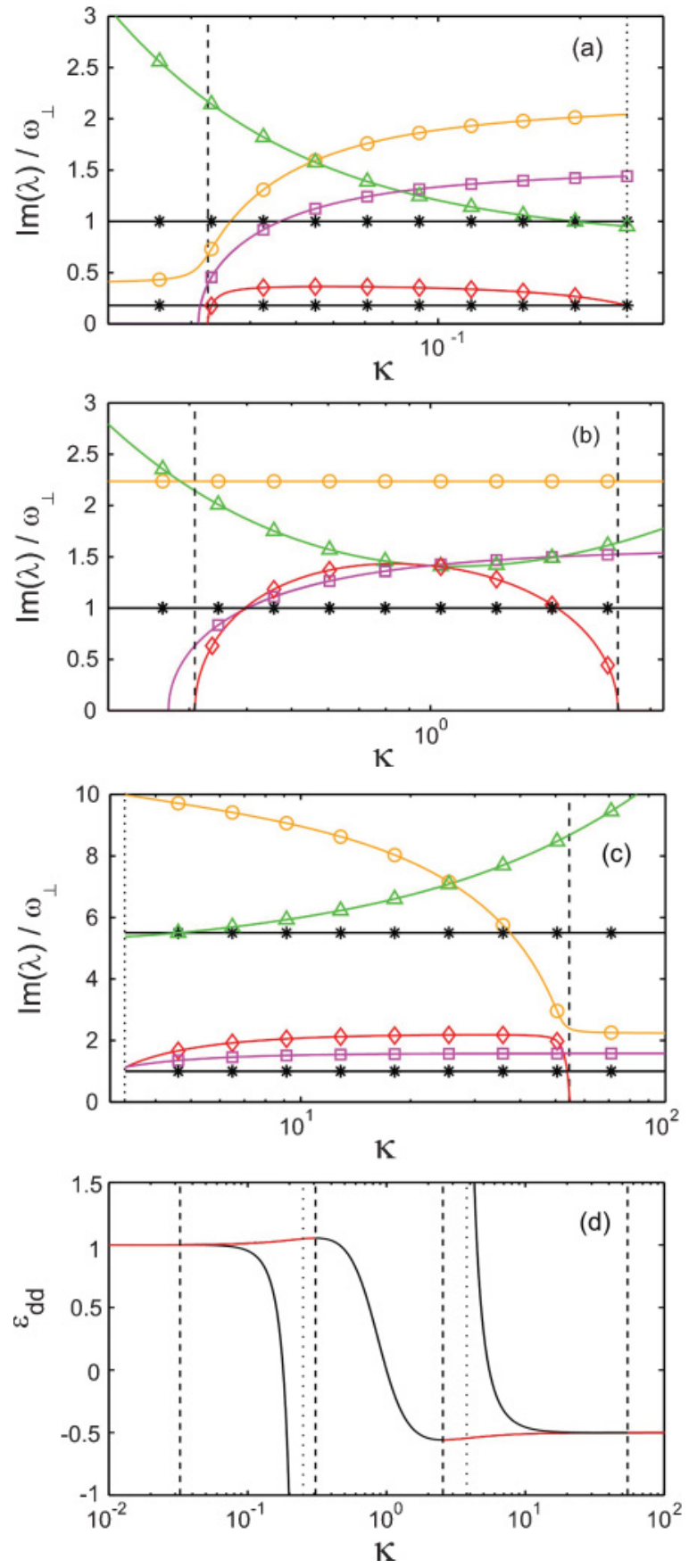

FIG. 5. (Color online) Excitation frequencies as a function of condensate aspect ratio $\kappa$ for a cylindrically-symmetric trap with aspect ratio (a) $\gamma=0.18$, (b) $\gamma=1$, and (c) $\gamma=5.5$. Shown are the results for the modes $M$ (orange, circles), $D$ (black, stars), $Q_{1}$ (red, diamonds), $Q_{2}$ (purple, squares), and $\mathrm{Sc}_{x z}$ ( $=\mathrm{Sc}_{y z}$ ) (green, triangles). (d) Static solutions $\kappa$ for $\gamma=0.18,1$, and 5.5. Vertical dashed lines mark the transition from stable to unstable for the static solution, and this coincides with the point at which one of the frequencies tends to zero. Vertical dotted lines mark the point at which the static solution ceases to exist altogether.

vary rapidly as a function of $\varepsilon_{\mathrm{dd}}$, but much more smoothly as a function of $\kappa$, and so it is easier to view the behavior as a function of $\kappa$. For completeness we have included the corresponding plot of the frequencies, but as a function of $\varepsilon_{\mathrm{dd}}$, 
in Appendix A. Also, analytic expressions for the frequencies of the $M$ and $Q_{1}$ modes in a cylindrically symmetric dipolar $\mathrm{BEC}$ in the TF regime can be found in [26].

It is worth pointing out that the condensate shape accounts for a significant part of the physics of these systems, and so $\kappa$ is a good variable to work with. For example, in the problem of a rotating dipolar BEC, the critical rotation frequency at which a vortex becomes energetically favorable is exactly the same as that in a purely $s$-wave BEC providing one corrects for the change in the aspect ratio due to the dipolar interactions [72]. However, $\kappa$ alone does not contain all the physics. In the case of the calculation of the excitation frequencies, this is clear from Eq. (25) which depends upon both $\left(\nabla \cdot n_{\mathrm{eq}} \nabla\right) \delta S$ and $\varepsilon_{\mathrm{dd}} K \delta n$. The former term has a direct dependence upon $\kappa$ via the equilibrium density profile $n_{0}(\mathbf{r})$, whereas the latter term does not.

We consider three values of trap ratio $\gamma$, which fall into three distinct regimes: (1) $\gamma<\gamma_{\text {crit }}^{-}$, (2) $\gamma_{\text {crit }}^{-}<\gamma<\gamma_{\text {crit }}^{+}$, and (3) $\gamma_{\text {crit }}^{+}<\gamma$. Recall that $\gamma_{\text {crit }}^{+}\left(\gamma_{\text {crit }}^{-}\right)$is the critical value above (below) which there exist stable solutions for $\varepsilon_{\mathrm{dd}} \rightarrow$ $+\infty(-\infty)$, see also Fig. 2. In each case, the aspect ratio of the stable solutions exists over a finite range $\kappa=\left[\kappa^{-}, \kappa^{+}\right]$. We will now discuss each regime in turn.

\section{1. $\gamma<\gamma_{\text {crit }}^{-}$}

In Fig. 5(a) we present the excitation frequencies for $\gamma=0.18$ as a function of $\kappa$. The corresponding static solutions are shown as the left-hand curve in Fig. 5(d) and confirm that the stable static solutions (solid black part of curve) exist only over a range of $\kappa=\left[\kappa^{-}, \kappa^{+}\right]$, with $\kappa^{-} \approx 0.03$ and $\kappa^{+} \approx 0.25$ indicated by vertical lines (dashed and dotted, respectively). For $\kappa>\kappa^{+}$, no static solutions exist and so the excitation frequencies are not plotted beyond this point [dotted vertical line in Fig. 5(a) and leftmost dotted vertical line in 5(d)]. For $\kappa<\kappa^{-}$, the static solution is no longer a local energy minimum but becomes instead a saddle point or maximum that is unstable to collapse [transition marked with dashed, vertical line in Fig. 5(a) and leftmost dashed vertical line in 5(d)]. Although this solution is not stable we can still determine its excitation spectrum. Crucially, this will reveal which modes are responsible for collapse and which remain stable throughout.

Three dipole modes (stars) exist. Dipole modes, in general, are decoupled from the internal dynamics of the condensate [3] and are determined by the trap frequencies $\omega_{x}, \omega_{y}$, and $\omega_{z}$. This provides an important check on our code. For the cylindrically symmetric case, $\omega_{x}=\omega_{y}=\omega_{\perp}$, and hence only two distinct dipole modes are visible. For $\kappa<\kappa^{-}$the dipole frequencies remain constant, indicating the dynamical stability of this mode.

In general, the remaining modes vary with the dipolar interactions. Perhaps the key mode here is the quadrupole $Q_{1}$ mode (diamonds). At the point of collapse the $Q_{1}$ frequency decreases to zero. This is connected to the dynamical instability of this mode since $\operatorname{Re}(\lambda)>0$ for $\kappa<\kappa^{-}$. The physical interpretation of this is that the $Q_{1}$ mode, which comprises an anisotropic oscillation in which the condensate periodically elongates and then flattens, mediates the collapse of the condensate into an infinitely narrow cigar-shaped BEC. In the energy landscape picture, this occurs because the barrier between the local energy minimum and the collapsed
$R_{x, y}=0$ state disappears for $\kappa<\kappa^{-}$. The $Q_{2}$ quadrupole mode (squares) decreases to zero, and becomes dynamically unstable, after one passes into the unstable regime as indicated in Fig. 5(d). The monopole $M$ mode (circles) remains stable for $\kappa<\kappa^{-}$and increases with $\kappa$ above this point.

$$
\text { 2. } \gamma_{\text {crit }}^{-}<\gamma<\gamma_{\text {crit }}^{+}
$$

In Fig. 5(b) we present the excitation frequencies for $\gamma=1$ as a function of $\varepsilon_{\mathrm{dd}}$. Since $\gamma_{\text {crit }}^{-}<\gamma<\gamma_{\text {crit }}^{+}$, the solutions exist over a finite range of $\varepsilon_{\mathrm{dd}}$. In terms of $\kappa$, collapse occurs at both limits of its range, i.e., for $\kappa<\kappa^{-}$and $\kappa>\kappa^{+}$, where $\kappa^{-} \approx$ 0.3 and $\kappa^{+} \approx 2.5$ [dashed vertical lines in Figs. 5(b) and 5(d)].

Since the trap is spherically symmetric, the dipole modes (stars) all have identical frequency, i.e., $\omega_{\perp}$. The $Q_{1}$ quadrupole frequency (diamonds) decreases to zero at both points of collapse, $\kappa^{-}$and $\kappa^{+}$. In the former case, this corresponds to the anisotropic collapse into an infinitely narrow BEC, while in the latter case, collapse occurs into an infinitely flattened BEC. In the low $\kappa$ regime, the $Q_{2}$ quadrupole mode (squares) becomes unstable just past the point of collapse, but shows no instability in the opposite limit for $\kappa>\kappa^{+}$.

It is interesting to note that the monopole mode (circles) shows no dependence on $\kappa$ and therefore the dipolar interactions, in agreement with [26]. Additionally, we find that the aspect ratio of the density perturbation remains fixed at precisely 1 for all values of the condensate aspect ratio $\kappa$. These observations are specific to the case of $\gamma=1$.

$$
\text { 3. } \gamma>\gamma_{\text {crit }}^{+}
$$

In Fig. 5(c) we plot the excitation frequencies for $\gamma=$ 5.5. For $\kappa<\kappa^{-}$, no static solutions exist, and for $\kappa>\kappa^{+}$, no stable solutions exist. Here $\kappa^{-} \approx 3.3$ and $\kappa^{+} \approx 54$ [dotted and dashed vertical lines, respectively, in Figs. 5(c) and 5(d)].

Again, the dipole modes are constant, while the remaining modes vary with dipolar interactions. Apart from the quadrupole $Q_{1}$ mode, all modes are stable past the point of collapse, including the $Q_{2}$ quadrupole mode. The $Q_{1}$ mode decreases to zero at the point when the condensate collapses to an infinitely flattened pancake BEC, which is again consistent with this mode mediating the anisotropic collapse.

In real experiments with a finite number of atoms, the zeropoint kinetic energy can be expected to extend the region of stability of the BEC. Therefore, in systems that deviate from the TF limit, we expect the $Q_{1}$ frequency to go to zero at a smaller value of $\kappa$ than that shown in Fig. 5(a), to go to zero at smaller and larger values of $\kappa$ at the left- and right-hand sides, respectively, than those shown in Fig. 5(b), and to go to zero at a larger value of $\kappa$ than shown in Fig. 5(c). In this context, we note that previous calculations of collective excitations in dipolar BECs by Góral and Santos [25] using a Gaussian ansatz (which takes account of zero-point energy and so is expected to be more accurate close to collapse but less accurate in the TF regime) also found that instabilities of the $Q_{1}$ mode were responsible for collapse when $g>0$.

\section{B. Variation with dipolar interactions $\varepsilon_{\mathrm{dd}}$ for $\boldsymbol{g}<\mathbf{0}$}

We now consider the analogous case but with $g<0$. As shown in Sec. II C, stable solutions only exist for $\gamma>\gamma_{\text {crit }}^{+}=$ 5.17 and $\gamma<\gamma_{\text {crit }}^{-}=0.19$, with no stable solutions existing in 

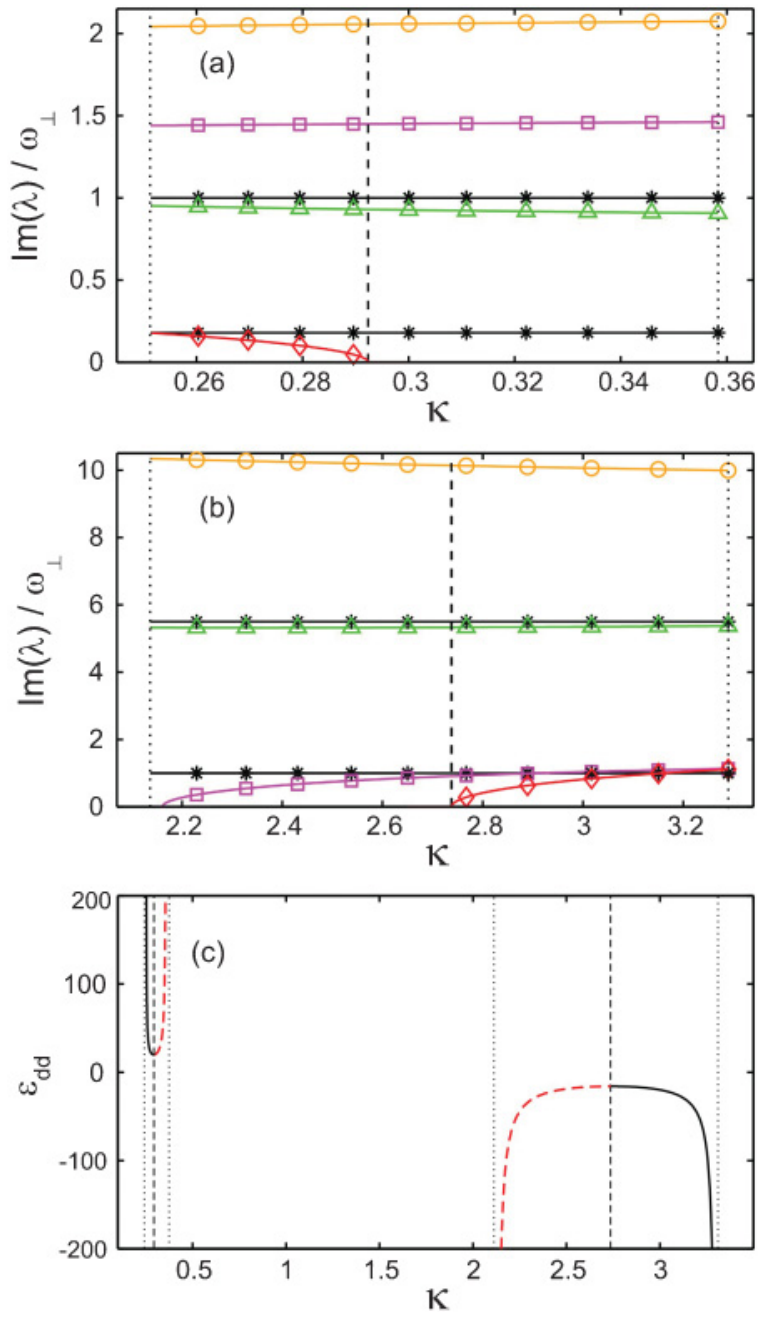

FIG. 6. (Color online) Excitation frequencies as a function of condensate aspect ratio $\kappa$ for a $g<0$ cylindrically symmetric trap with aspect ratio (a) $\gamma=0.18$ and (b) $\gamma=5.5$, with corresponding static solutions shown in (c). Included are the results for the modes $M$ (orange, circles), $D$ (black, stars), $Q_{1}$ (red, diamonds), $Q_{2}$ (purple, squares), and Sc (green, triangles). Dashed vertical lines indicate the critical point at which the stable static solutions turn into unstable ones, dotted vertical lines indicate endpoints of branches where static solutions cease to exist.

the range $\gamma_{\text {crit }}^{-}<\gamma<\gamma_{\text {crit. }}^{+}$. Hence we will only consider the two regimes of (1) $\gamma<\gamma_{\text {crit }}^{-}$and (2) $\gamma>\gamma_{\text {crit }}^{+}$.

\section{1. $\gamma<\gamma_{\text {crit }}^{-}$}

In Fig. 6(a) we present the excitation frequencies in a highly elongated trap $\gamma=0.18$. Stable static solutions exist only for $\kappa^{-}<\kappa<\kappa^{+}$where $\kappa^{-} \approx 0.25$ and $\kappa^{+} \approx 0.29$. In this regime we find that all collective frequencies are purely imaginary and finite, and therefore stable. At the critical point for collapse $\kappa \approx 0.29$ the $Q_{1}$ mode frequency passes through zero and becomes purely real, signifying its dynamical instability. This shows that, as for $g>0$, the $Q_{1}$ mode mediates collapse, and therefore collapse proceeds in a highly anisotropic manner due to the anisotropic character of the dipolar interactions. The remaining modes do not become dynamically unstable past the critical point, and only vary weakly over the range of $\kappa$ shown. It should also be remarked that higher-order modes with polynomial degree $v>2$ also become unstable within the range $\kappa^{-}<\kappa<\kappa^{+}$where no stable parabolic solutions lie, further highlighting the metastability of the $g<0$ states and confirming the relevance of the predictions made by the uniform-density Bogoliubov spectrum (20) for a system in the TF regime.

$$
\text { 2. } \gamma>\gamma_{\text {crit }}^{+}
$$

Figure 6(b) shows the mode frequencies in a highly flattened trap $\gamma=5.5$, for which stable static solutions exist only in the regime $\kappa^{-}<\kappa<\kappa^{+}$where $\kappa^{-} \approx 2.7$ and $\kappa^{+} \approx 3.3$. Similarly, at the point of collapse $\kappa \approx 2.7$ the $Q_{1}$ mode has zero frequency and is dynamically unstable. Well below the critical point the $Q_{2}$ mode frequency also becomes zero and dynamically unstable.

\section{Variation with trap ratio $\gamma$}

Having illustrated in the previous section how the excitation frequencies behave for $g<0$, from now on we will limit ourselves to the case of $g>0$. In Fig. 7 we plot the excitation frequencies as a function of $\gamma$ for various values of $\varepsilon_{\mathrm{dd}}$. A common feature is that the dipole frequencies scale with their corresponding trap frequencies, such that $\omega_{D_{x}}=\omega_{D_{y}}=\omega_{\perp}$ and $\omega_{D_{z}}=\gamma \omega_{\perp}$. We now consider the three regimes of zero, negative, and positive $\varepsilon_{\mathrm{dd}}$.

\section{1. $\varepsilon_{\mathrm{dd}}=0$}

For $\varepsilon_{\mathrm{dd}}=0$ stable solutions exist for all $\gamma$ and the corresponding mode frequencies are plotted in Fig. 7(a). Our results agree with previous studies of nondipolar BECs where analytic expressions for the mode frequencies can be obtained, see, e.g., [2] and [3]. The $Q_{2}$ quadrupole mode has fixed frequency $\omega_{Q_{2}}=\sqrt{2} \omega_{\perp}$. The scissors-mode frequency corresponds to $\omega_{S c_{x z}}=\omega_{S c_{y z}}=\sqrt{1+\gamma^{2}} \omega_{\perp}$, and the remaining modes obey the equation [3]

$$
\omega^{2}=\omega_{\perp}^{2}\left(2+\frac{3}{2} \gamma^{2} \pm \frac{1}{2} \sqrt{16-16 \gamma^{2}+9 \gamma^{4}}\right),
$$

where the "+" and "-" solutions correspond to $\omega_{M}$ and $\omega_{Q_{1}}$, respectively.

\section{2. $\varepsilon_{\mathrm{dd}}<0$}

For $\varepsilon_{\mathrm{dd}}=-0.75$ [Fig. 7(b)], stable solutions and collective modes exist up to a critical trap ratio $\gamma^{\max } \approx 0.56$. Beyond that, the attractive nature of side-by-side dipoles (recall $C_{\mathrm{dd}}<0$ ) makes the system unstable to collapse.

For all of the modes except the $Q_{1}$ quadrupole mode we see the same qualitative behavior as for the nondipolar case (gray lines) with the modes extending right up to the point of collapse with no qualitative distinction from the nondipolar case. The $Q_{1}$ quadrupole mode, on the other hand, initially increases with $\gamma$, like the nondipolar case, but as it approaches the point of collapse, it rapidly decreases toward zero. Above $\gamma^{\max }$, the $Q_{1}$ mode is dynamically unstable. 

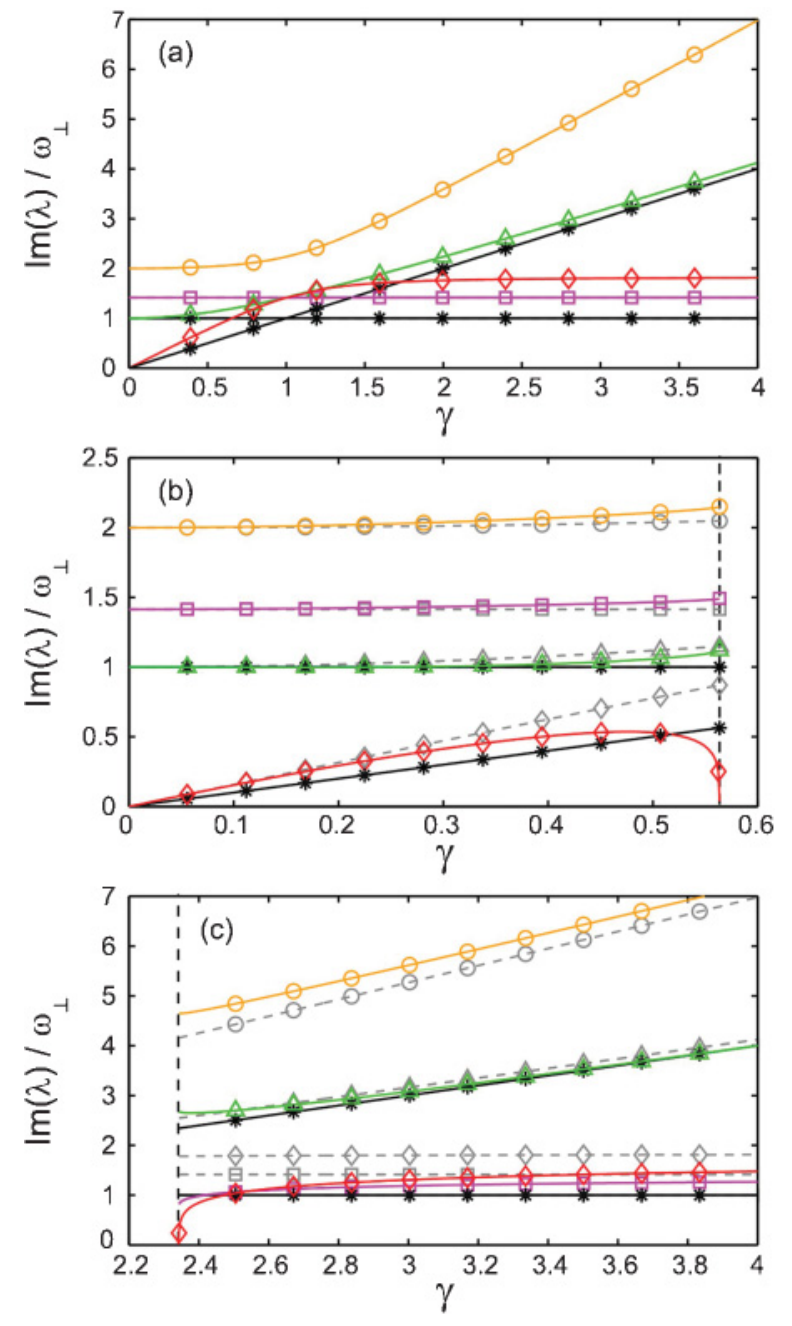

FIG. 7. (Color online) Excitation frequencies in a cylindrically symmetric trap as a function of the trap aspect ratio $\gamma$ for (a) $\varepsilon_{\mathrm{dd}}=0$, (b) $\varepsilon_{\mathrm{dd}}=-0.75$, and (c) $\varepsilon_{\mathrm{dd}}=1.5$. Shown are the results for the modes $M$ (orange, circles), $D$ (black, stars), $Q_{1}$ (red, diamonds), $Q_{2}$ (purple, squares), and Sc (green, triangles). In (b) and (c) the frequencies for $\varepsilon_{\mathrm{dd}}=0$ are included as dashed, gray lines.

\section{3. $\varepsilon_{\mathrm{dd}}>\mathbf{0}$}

For $\varepsilon_{\text {dd }}=1.5$ [Fig. 7(c)], stable solutions exist only above a lower critical trap ratio $\gamma^{\mathrm{min}} \approx 2.3$. For $\gamma<\gamma^{\text {min }}$ the attraction of the end-to-end dipoles becomes dominant and induces collapse. Indeed, we find that the frequency of the $Q_{1}$ mode passes through zero and is dynamically unstable for $\gamma<\gamma^{\mathrm{min}}$. Above this, the $Q_{1}$ and $Q_{2}$ frequencies increase toward the limiting values of the nondipolar frequencies of $1.82 \omega_{\perp}$ and $\sqrt{2} \omega_{\perp}$ because in a very pancake-shaped trap the atoms cannot sample the anisotropy of the interactions. The remaining modes behave qualitatively like the nondipolar modes for $\gamma>\gamma^{\text {min }}$.

\section{EXCITATIONS IN A NON-CYLINDRICALLY-SYMMETRIC TRAP AND RELEVANCE TO ROTATING-TRAP SYSTEMS}

In this section we will apply our approach to the most general case of non-cylindrically-symmetric traps. An important experimental scenario where this occurs is when condensates are rotated in elliptical harmonic traps. This has provided a robust method for generating vortices and vortex lattices in condensates (see Ref. [73] for a review). While the trap ellipticity in the $x-y$ plane is typically small (in most experiments it is of the order of a few percent), the rotation accentuates the ellipticity induced in the condensate. Indeed, one can derive effective harmonic trap frequencies for the condensate which show that the effective ellipticity can be orders of magnitude greater than the static ellipticity $[35,42,44]$.

The $Q_{2}$ mode can be pictured as a surface wave traveling around the edge of the condensate. It has a similar shape to the rotating elliptical deformation of the trap, and when the trap is rotated at frequencies close to that of the $Q_{2}$ mode then even a perturbatively small trap deformation strongly couples to this mode. When viewed from the frame of reference rotating with the trap, the excitation of the $Q_{2}$ mode appears as a bifurcation of the stationary condensate into a new stationary state which mixes in some of the $Q_{2}$ mode, and the condensate therefore develops an elliptical shape in the $x-y$ plane. For some ranges of rotation speeds this new stationary state is in turn dynamically unstable to the excitation of higher order modes [35,36,42]. This dynamical instability disrupts the condensate and is the first step in the process by which vortices enter. Although this process is complex, the dynamical instability that initiates it is accurately described within the TF approximation because the modes which are initially excited are of sufficiently long wavelength. The predictions obtained within the TF approximation are in excellent agreement with both experiments [45] and numerical simulations of the GPE $[43,46]$. Although we will not specifically consider rotation further here, our methodology can be easily extended to this scenario [35].

As in Sec. II D, we consider finite trap ellipticity $\epsilon$ in the $x-y$ plane. In Fig. 8 we present the mode frequencies as a function of ellipticity $\epsilon$ for three different examples. There are some important generic differences to the cylindrical case. Due to the complete anisotropy of the trapping potential, the dipole mode frequencies (stars) all differ and are equal to the corresponding trap frequencies $\omega_{x}=\sqrt{1-\epsilon} \omega_{\perp}, \omega_{y}=\sqrt{1+\epsilon} \omega_{\perp}$, and $\omega_{z}=$ $\gamma \omega_{\perp}$. The monopole mode is present (circles) and its frequency increases with $\epsilon$. Strictly speaking the $Q_{2}$ mode is no longer present due to the breakdown of cylindrical symmetry. Instead we find a new $Q_{1}$ mode appearing (upper diamonds) which corresponds to the $Q_{1}^{y z}$ mode for $\varepsilon_{\mathrm{dd}}>0$ and the $Q_{1}^{x z}$ mode for $\varepsilon_{\mathrm{dd}} \leqslant 0$. The usual quadrupole mode $Q_{1}^{x y}$ is also present. The reader is reminded that the superscript in the $Q_{1}$ mode notation refers to the in-phase radii, the remaining radius oscillates out of phase with the other two. Although there are actually three permutations of $Q_{1}$, only two appear for any given value of $\varepsilon_{\mathrm{dd}}$ since linear combinations of these and the monopole mode can form the remaining $Q_{1}$ mode.

We will now consider the specific features for the cases presented in Fig. 8. For $\varepsilon_{\mathrm{dd}}=0$ and $\gamma=1$ [Fig. 8(a)], the solutions are stable right up to $\epsilon=1$. At this limit the $x$ direction becomes untrapped, and this causes the system to become unstable with respect to the dipole $D_{x}$ mode, as well the $Q_{1}^{x y}$ mode which can now expand freely along the $x$ axis. For $\varepsilon_{\mathrm{dd}}=-0.6$ and $\gamma=0.8$ [Fig. 8(b)], the solutions become 

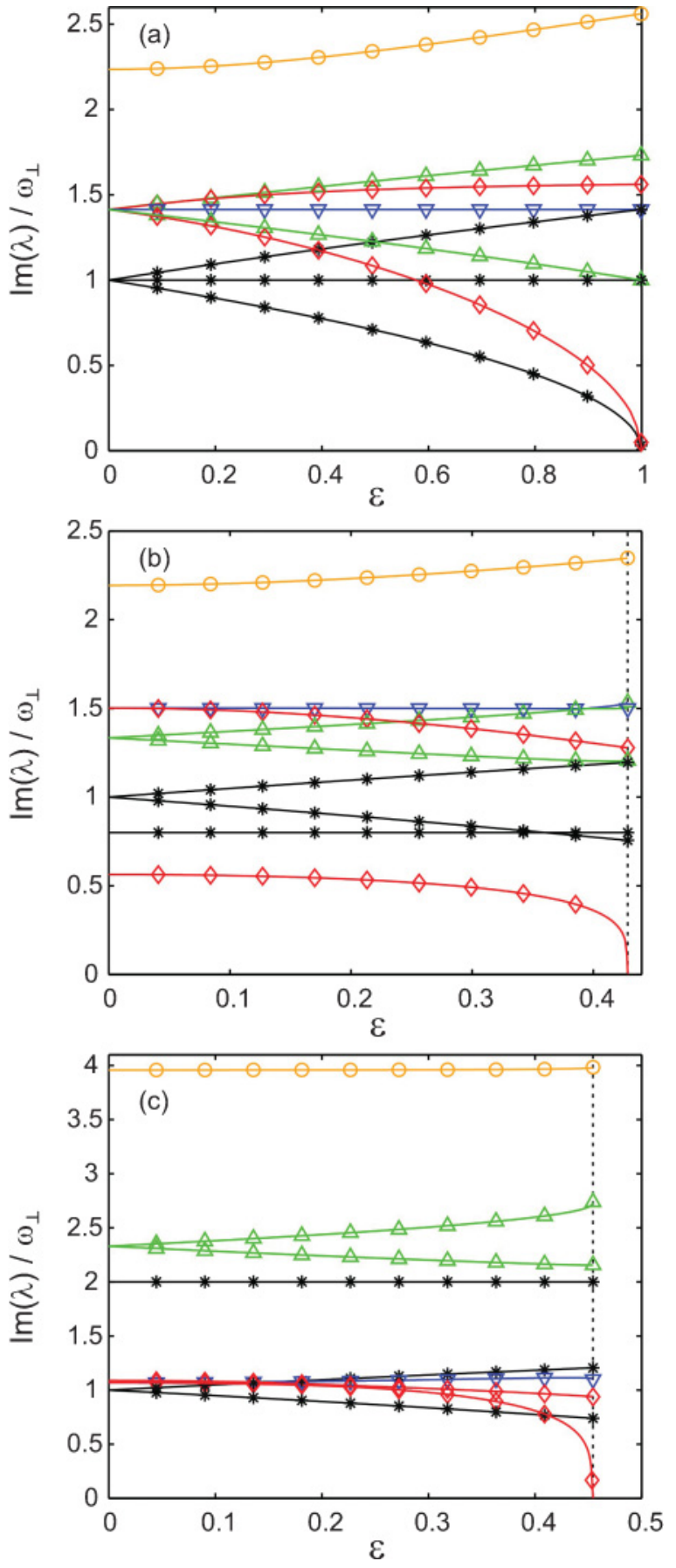

FIG. 8. (Color online) Excitation frequencies in a noncylindrically-symmetric trap as a function of the trap ellipticity $\epsilon$ in the $x-y$ plane for the cases of (a) $\varepsilon_{\mathrm{dd}}=0$ and $\gamma=1$, (b) $\varepsilon_{\mathrm{dd}}=$ -0.6 and $\gamma=0.8$, and (c) $\varepsilon_{\mathrm{dd}}=1.25$ and $\gamma=2$. Shown are the modes $D$ (black, stars), $M$ (orange, circles), $Q_{1}$ (red, diamonds), $\mathrm{Sc}_{x y}$ (blue, triangles pointing down), $\mathrm{Sc}_{y z}$ (green, triangles pointing up, upper branch), and $\mathrm{Sc}_{x z}$ (green, triangles pointing up, lower branch).

unstable to collapse at $\epsilon \approx 0.425$. Only the lower $Q_{1}^{x y}$ mode becomes dynamically unstable at this point, indicating that it is the mode responsible for collapse, which is toward a pancakeshaped system. For $\varepsilon_{\mathrm{dd}}=1.25$ and $\gamma=0.2$ [Fig. 8(c)], the solutions become unstable to collapse at $\epsilon \approx 0.45$. We again observe that the same $Q_{1}$ mode mediates the collapse, only this time the collapse is toward a cigar-shaped system. The other modes remain stable.

\section{SCISSORS MODES}

In this section, we study the so-called scissors modes of the condensate, which describe an oscillatory rotation of the condensate in response to a sudden rotation of an elliptic trap about a small angle. The importance of the scissors modes is that they clearly distinguish between superfluid and nonsuperfluid systems and can thus be used to gain information about the nature of the quantum state of a trapped ultracold gas [38]. The special relevance of this to ultracold dipolar Bose gases is that when the attractive portion of the dipolar interactions becomes important, such as in cigar-shaped systems aligned along the external polarizing field, it is possible that simple Bose-Einstein condensation (macroscopic occupation of a single quantum state) is no longer energetically favored. Some time ago $[74,75]$, it was realized that due to quantum exchange effects, repulsive interactions in spatially uniform Bose gases favor simple Bose-Einstein condensation over fragmented Bose-Einstein condensation (macroscopic occupation of two or more quantum states) [76]. The converse is expected to be true in the presence of attractive interactions, although attractive interactions in a uniform system lead to mechanical collapse. The experimental realization of trapped ultracold gases with attractive $s$-wave interactions [65] was therefore a significant event: fragmentation could potentially be studied in systems with attractive interactions which are stabilized by their zero-point energy. However, the consensus now seems to be that in attractive $s$-wave systems, the magnitude of the interactions required to see significant fragmentation is too great for the zero-point energy to stabilize the system [77]. Dipolar interactions, on the other hand, are partially attractive and partially repulsive, the net balance being tunable via the shape of the atomic cloud. Recent work by Bader and Fischer [78] suggests that fragmentation can occur before collapse in low-dimensional trapped systems with anisotropic interactions. A comprehensive investigation of fragmentation in dipolar BECs is beyond the scope of the current paper, but below we take a step in this direction by calculating the properties of scissors modes of a dipolar BEC.

Experimentally, one of the simplest indicators of whether or not an atomic cloud is Bose-Einstein condensed is to examine the momentum distribution following free expansion after the trap is turned off [1]. For example, according to the equipartition theorem, a gas at thermal equilibrium will expand isotropically even if the trap was anisotropic. This is not true for a BEC which, due to its zero-point energy, expands most rapidly in the direction which was most tightly confined. However, for dipolar BECs the situation is complicated by the anisotropy of the long-range interactions which continue to act at some level even as the gas expands [8,9]. Quantized vortices are another "smoking gun" indicating the presence of a BEC, but these are not easy to controllably generate in the cigar-shaped systems which would be of primary interest (although they might be useful in cases where $C_{\mathrm{dd}}<0$, for which pancake-shaped BECs have dominant attractive interactions). Furthermore, in cigar-shaped systems 
with $C_{\mathrm{dd}}>0$, the rotation speed at which a vortex becomes energetically favorable diverges as $\varepsilon_{\mathrm{dd}}$ increases [72]. Scissors modes, on the other hand, offer an alternative vehicle for the investigation of superfluidity in dipolar systems which does not suffer from the difficulties mentioned above.

A detailed account of the scissors mode in a pure $s$-wave BEC can be found in [38]. The scissors mode of a trapped atomic cloud (thermal or Bose-Einstein condensed) is excited by suddenly rotating the anisotropic trapping potential over a small angle. Consequently, the atomic cloud will experience a restoring force exerted by the trap; and provided the angle of rotation is small, it will exhibit a shape-preserving oscillation around the new equilibrium position. The exact response of the atomic cloud to the torque of the rotated trapping potential depends strongly on the moment of inertia of the cloud. Since a superfluid is restricted to irrotational flow, it will have a significantly different moment of inertia compared to a thermal cloud. In particular, when the trap anisotropy vanishes, the moment of inertia of a superfluid also vanishes, whereas in a thermal cloud this is not the case. The superfluid scissors-mode frequency will consequently approach a finite value, whereas in a thermal cloud it will vanish as the trap anisotropy approaches zero [38]. A measurement of the scissors-mode frequency therefore constitutes a direct test for superfluidity $[3,38]$, as has been verified experimentally for nondipolar BECs [39,41].

In the following, we will consider the scissors mode to be excited by rotating the trapping potential as well as the external aligning field of the dipoles (except for the $\mathrm{Sc}_{x y}$ mode which does not require a rotation of the external field) simultaneously and abruptly through a small angle, such that the condensate suddenly finds itself in a rotationally displaced configuration. Three scissors modes now appear due to the three distinct permutations of this mode, namely, $\mathrm{Sc}_{x y}$ (triangles pointing down in Fig. 8), $\mathrm{Sc}_{y z}$ (triangles pointing up), and $\mathrm{Sc}_{x z}$ (triangles pointing up). Clearly, from Fig. 8, the oscillation frequencies of the scissors modes are affected by the dipolar interactions. The effect of the dipolar interactions is twofold. First, since the dipolar interactions change the aspect ratio of the condensate, both the moment of inertia of the condensate and the torque from the trapping potential acting on it will be altered, which will consequently alter the oscillation frequency. Second, for the $\mathrm{Sc}_{x z}$ and $\mathrm{Sc}_{y z}$ modes, there is an additional force present which is related to the relative position of the dipoles. This effect is most easily understood when considering a cigar-shaped condensate. When such a condensate is rotated with respect to the aligning field, the dipoles are on average slightly more side-by-side than in the equilibrium situation. As a result, there will be a dipolar restoring force trying to realign the dipoles, which in turn is expected to affect the scissors-mode frequencies. Figure 9 schematically illustrates this process for the $\mathrm{Sc}_{x z}$ mode. For a pancake-shaped condensate, the effect is opposite. Since the dipolar interaction potential is rotationally invariant in the $x-y$ plane, the dipolar restoring force is absent for the $\mathrm{Sc}_{x y}$ mode.

Explicit expressions for the scissors frequencies can be obtained by performing the procedure outlined in Sec. III analytically rather than numerically. We start with the frequency $\omega_{s x y}$ of the $\mathrm{Sc}_{x y}$ mode, in which case we only expect an influence of dipolar interactions through changes in the geometry,

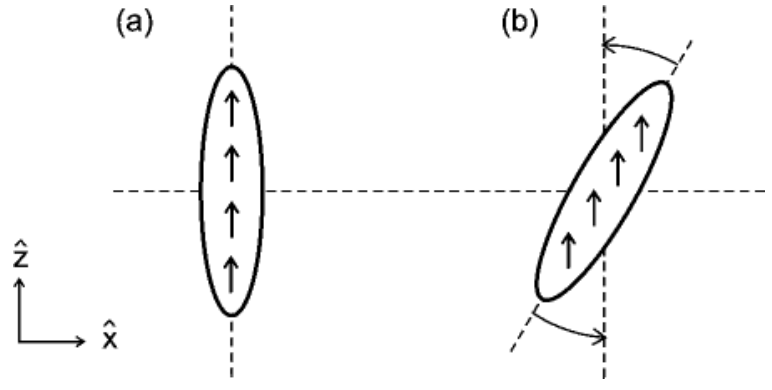

FIG. 9. Schematic illustration of the dipolar restoring force for the $\mathrm{Sc}_{x z}$ mode. When the condensate is rotated with respect to the dipole alignment axis $\hat{z}$ as in situation (b), the dipoles will on average be more side-by-side than in the aligned case, situation (a). Since this is an energetically unfavorable configuration compared to the aligned case, there will consequently be a dipolar restoring force present in (b) trying to realign the condensate, illustrated by arrows.

and find

$$
\omega_{s x y}^{2}=2 \omega_{\perp}^{2} \epsilon\left(\frac{\kappa_{x}^{2}-\kappa_{y}^{2}}{\kappa_{y}^{2}+\kappa_{x}^{2}}\right)^{-1},
$$

where it should be noted that the quantity in brackets is precisely the ellipticity of the condensate. The $\mathrm{Sc}_{x y}$ frequency does not depend explicitly on the strength of the dipolar interactions $\varepsilon_{\mathrm{dd}}$, but merely on the condensate ellipticity, which is an indication of the absence of a dipolar restoring force as discussed above. The condensate ellipticity turns out to be approximately proportional to the trap ellipticity, where the constant of proportionality is dependent on the dipolar interaction strength $\varepsilon_{\mathrm{dd}}$ and axial trapping strength $\gamma$. As a result, the $\mathrm{Sc}_{x y}$ scissors frequencies shown in Fig. 8 are (almost) independent of the trap ellipticity for fixed values of $\varepsilon_{\mathrm{dd}}$ and $\gamma$. Figure 10(a) shows the $\mathrm{Sc}_{x y}$ frequency as a function of the axial trapping strength $\gamma$, for various dipolar interaction strengths $\varepsilon_{\mathrm{dd}}$. In the presence of dipolar interactions, the condensate ellipticity deviates from the trap ellipticity $\epsilon$ (see Sec. II D), and hence the $\mathrm{Sc}_{x y}$ frequency also changes when dipolar interactions are switched on. In the absence of dipolar interactions [dashed line in Fig. 10(a)], the trap and condensate ellipticity are equal, and the $\mathrm{Sc}_{x y}$ frequency is independent of the condensate size, trap ellipticity, as well as the $s$-wave interaction strength [38]. For very prolate $(\gamma \ll 1)$ and very oblate $(\gamma \gg 1)$ traps, the dipolar interactions become either mainly attractive or mainly repulsive and lose their anisotropic character. The dipolar potential becomes contactlike and can be renormalized into the $s$-wave interactions (see Sec. IIC or [34]), which do not influence the scissors-mode frequency. This effect is visible in Fig. 10(a) in the form of the scissors frequency returning to the nondipolar value for extremal values of $\gamma$. Finally, we would like to point out a remarkable similarity between the scissors frequencies shown in Fig. 10(a) and the trap rotation frequencies at which the static solution diagram of a rotating dipolar BEC shows a bifurcation point, as investigated in Ref. [36] [see Fig. 1(b) therein]. For all values of $\gamma$ and $\varepsilon_{\mathrm{dd}}$ the scissors frequency is precisely twice the bifurcation frequency. Presumably, the underlying connection is the fact that the scissors mode $\mathrm{Sc}_{x y}$ has the same superfluid field as a stationary state of a BEC in a rotating trap. 

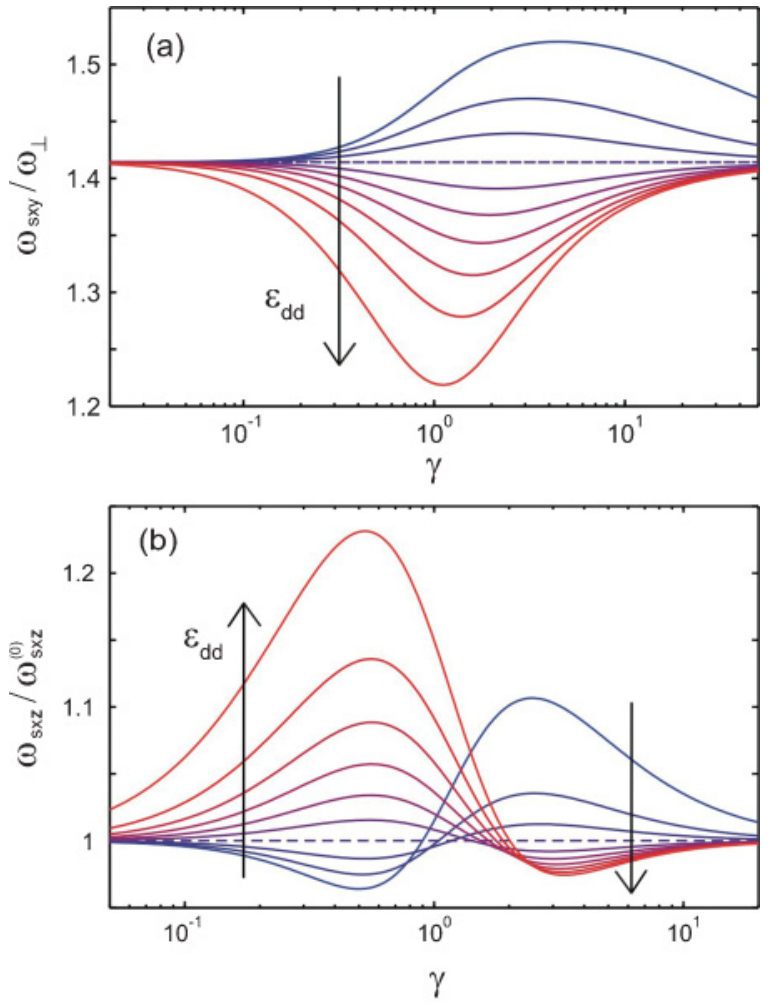

FIG. 10. (Color online) Scissors frequencies as a function of the axial trapping strength $\gamma$ for various values of $\varepsilon_{\mathrm{dd}}$, with $-0.45 \leqslant$ $\varepsilon_{\mathrm{dd}} \leqslant 0.9$ and increasing in the direction of the arrow in steps of 0.15 . The dashed line indicates $\varepsilon_{\mathrm{dd}}=0$. (a) Frequency of $\mathrm{Sc}_{x y}$ mode for fixed trap ellipticity of $\epsilon=0.1$. For very prolate $(\gamma \ll 1)$ or very oblate $(\gamma \gg 1)$ systems, the dipolar interactions renormalize into the $s$-wave interactions and $\omega_{s x y}$ returns to the nondipolar value. (b) Frequency $\omega_{s x z}$ of the $\mathrm{Sc}_{x z}$ mode as a function of $\gamma$ for a cylindrically symmetric trap, scaled to the nondipolar frequency $\omega_{s x z}^{(0)}$.

Turning our attention to the $\mathrm{Sc}_{x z}$ and $\mathrm{Sc}_{y z}$ frequencies, the analytical calculation yields

$$
\begin{aligned}
& \frac{\omega_{s x z}^{2}}{\omega_{z}^{2}}=\left(\frac{1}{\kappa_{x}^{2}}+\frac{1}{\kappa_{y}^{2}}\right) \frac{1-\varepsilon_{\mathrm{dd}}\left(1-\frac{9}{2} \kappa_{x}^{3} \kappa_{y} \beta_{102}\right)}{1-\varepsilon_{\mathrm{dd}}\left(1-\frac{9}{2} \kappa_{x} \kappa_{y} \beta_{002}\right)}, \\
& \frac{\omega_{s y z}^{2}}{\omega_{z}^{2}}=\left(\frac{1}{\kappa_{x}^{2}}+\frac{1}{\kappa_{y}^{2}}\right) \frac{1-\varepsilon_{\mathrm{dd}}\left(1-\frac{9}{2} \kappa_{x} \kappa_{y}^{3} \beta_{012}\right)}{1-\varepsilon_{\mathrm{dd}}\left(1-\frac{9}{2} \kappa_{x} \kappa_{y} \beta_{002}\right)} .
\end{aligned}
$$

Here, the quantity $\varepsilon_{\mathrm{dd}}$ appears explicitly, and as such the frequencies depend directly on the strength of the dipolar interactions, an effect we attribute to the dipolar restoring force. Figure 10(b) shows the above frequencies for a cylindrically symmetric trap and as a function of the axial trapping strength $\gamma$, for various values of $\varepsilon_{\mathrm{dd}}$. There are two distinct effects to be noted. First, when $\varepsilon_{\mathrm{dd}}>0\left(\varepsilon_{\mathrm{dd}}<0\right)$ the scissors frequencies go up (down) for cigar-shaped systems and down (up) for pancake-shaped systems. This behavior is consistent with what one would expect in the presence of a dipolar restoring force. Second, for $\gamma \ll 1$ and $\gamma \gg 1$ we see that the $\omega_{s x z}$ frequency approaches the nondipolar value again. For the $\omega_{s x y}$ frequency this effect could be explained solely by the fact that for such values of $\gamma$ the condensate aspect ratios return to the nondipolar values. However, for the $\mathrm{Sc}_{x z}$ and $\mathrm{Sc}_{y z}$ modes we have to account for the apparent vanishing of the dipolar restoring force as well. To see why it plays no part here, we have to analyze the expectation values of the quantity $R=\sqrt{x^{2}+y^{2}+z^{2}}$. For $\gamma \ll 1$, we have $\langle R\rangle \simeq\langle|z|\rangle \rightarrow \infty$, and for $\gamma \gg 1$ we have $\langle R\rangle \simeq\left\langle\sqrt{x^{2}+y^{2}}\right\rangle \rightarrow \infty$. Although in both cases the torque exerted by the dipolar restoring force is proportional to $\langle R\rangle$ and in principle approaches infinity, it vanishes relative to the other two quantities contributing to the scissors frequencies, namely, the moment of inertia of the condensate and the torque exerted by the trap, which both scale as $\left\langle R^{2}\right\rangle$ [40]. In Fig. 10(b), this behavior can be observed for the extremal values of $\gamma$, where the scissors frequencies approach those of the nondipolar case.

\section{CONCLUSIONS}

In this paper we have performed an investigation into the static and dynamic states of trapped dipolar Bose-Einstein condensates in the Thomas-Fermi regime. We have extended our previous work in this area by examining new regimes of dipolar and $s$-wave interactions (namely, positive and negative values of $C_{\mathrm{dd}}$ and $g$ ), non-cylindrically-symmetric traps, and different classes of collective excitation, including the scissors modes. Our approach is based upon the analytic calculation of the nonlocal dipolar mean-field potential inside the condensate, for an arbitrary polynomial density profile, and exploiting the fact that this potential is again of polynomial form. Using this method, we have examined the stability of static states and collective excitations, including the behavior of the collective excitations as a function of the trap aspect ratio and ellipticity, and as a function of the relative strength of the dipolar and $s$-wave interactions. We consistently find that an instability of the $Q_{1}$ quadrupole mode mediates global collapse of a dipolar BEC whether $g>0$ or $g<0$. However, there are two critical trap ratios, $\gamma_{\text {crit }}^{+}=5.17$ and $\gamma_{\text {crit }}^{-}=0.19$, beyond which the BEC is stable against scaling fluctuations (monopole and quadrupole excitations) even as the strength of the dipolar interaction overwhelms the $s$-wave one, i.e., when $\varepsilon_{\mathrm{dd}} \rightarrow \pm \infty$. In the case of attractive $s$-wave interactions $(g<0)$, where the dipolar interactions can stabilize an otherwise unstable condensate, the magnetostriction seems to act counterintuitively (see Fig. 3), although upon closer examination the behavior can be explained by understanding how dipolar interactions behave in highly confined geometries.

We have paid special attention to the scissors modes because of their sensitivity to superfluidity, which we identify as an issue of particular interest in cigar-shaped dipolar condensates due to the possibility of fragmentation when the attractive part of the dipolar interaction becomes significant. Our expressions for the frequencies of the scissors modes include a term due to a restoring force which is not present in the pure $s$-wave case, and which we identify as arising due to an anisotropic dipolar realignment force. A freely available MATLAB implementation of the calculations outlined in this paper, including a graphical user interface, can be obtained online [52].

Note added. Recently a paper [79] has appeared announcing the results of an experiment measuring the collective excitations of a dipolar BEC. The authors state that they find good agreement with the results obtained previously 
by one of us in the Thomas-Fermi regime [26], of which the current work is an extension.

\section{ACKNOWLEDGMENTS}

We acknowledge support from The Netherlands Organisation for Scientific Research (NWO) (R.M.W.v.B. and S.J.J.M.F.K.), Canadian Commonwealth (N.G.P.), Australian Research Council (A.M.M.) and Natural Sciences and Engineering Research Council of Canada (D.H.J.O'D.). The authors also wish to thank T. Hortons for vital stimulation.

\section{APPENDIX A: COLLECTIVE MODE FREQUENCIES AS A FUNCTION OF $\varepsilon_{\mathrm{dd}}$}

In Sec. III we considered the effect of the dipolar interactions on the mode frequencies and plotted this as a function of $\kappa$ rather than $\varepsilon_{\mathrm{dd}}$ to remove the problem of the static solutions being double valued. However, since $\varepsilon_{\mathrm{dd}}$ is a more obvious experimental parameter, we have plotted the corresponding frequency plots of Fig. 5, but as a function of $\varepsilon_{\mathrm{dd}}$ in Fig. 11.

\section{APPENDIX B: CALCULATING THE DIPOLAR POTENTIAL INSIDE A HETEROGENOUS ELLIPSOIDAL BEC}

In this appendix we will concern ourselves with the calculation of integrals of the form

$$
\phi[\rho](\mathbf{r})=\frac{1}{4 \pi} \int \frac{\rho\left(\mathbf{r}^{\prime}\right)}{\left|\mathbf{r}^{\prime}-\mathbf{r}\right|} d x^{\prime} d y^{\prime} d z^{\prime},
$$

where the domain of integration is a general ellipsoid with semiaxes $R_{x}, R_{y}, R_{z}$, and the point $\mathbf{r}=(x, y, z)$ is an internal point of the ellipsoid. The square brackets indicate a functional dependence. When computing the dipolar potential in Eqs. (7), (8), and (26), we need to evaluate this integral in order to obtain the fictitious electrostatic potential $\phi\left[\rho_{i j k}\right](\mathbf{r})$ arising from a particle density of the form

$$
\rho_{i j k}=x^{i} y^{j} z^{k},
$$

with $i, j, k$ nonnegative integers. By taking linear combinations of the general term $\rho_{i j k}$ we can calculate the internal dipolar potential created by an arbitrary density distribution because Eq. (B1) defines a linear integral operator acting upon $\rho(\mathbf{r})$.

The physically relevant density distributions can naturally be divided into two classes:

(1) The inverted parabola $n(\mathbf{r})$ given by Eq. (10) which corresponds to the static ground state of the BEC.

(2) The excitations $\delta n(\mathbf{r})$ and $\delta S(\mathbf{r})$ given by Eq. (27) which can be written as linear combination of terms $x^{i} y^{j} z^{k}$.

Actually, these two classes have some overlap because certain low-lying excitations (monopole, quadrupole, and scissors modes), which would otherwise seem to fall into class 2 , can be described in terms of the parabolic density profile of class 1 but with time-oscillating radii (in the case of monopole and quadrupole modes) or time-oscillating symmetry axes (in the case of the scissors modes). In this appendix we give the general theory which works for all density distributions of the form (B2). In Appendix $\mathrm{C}$ we focus on the parabolic density distributions of class 1 , which is a particular case of the general

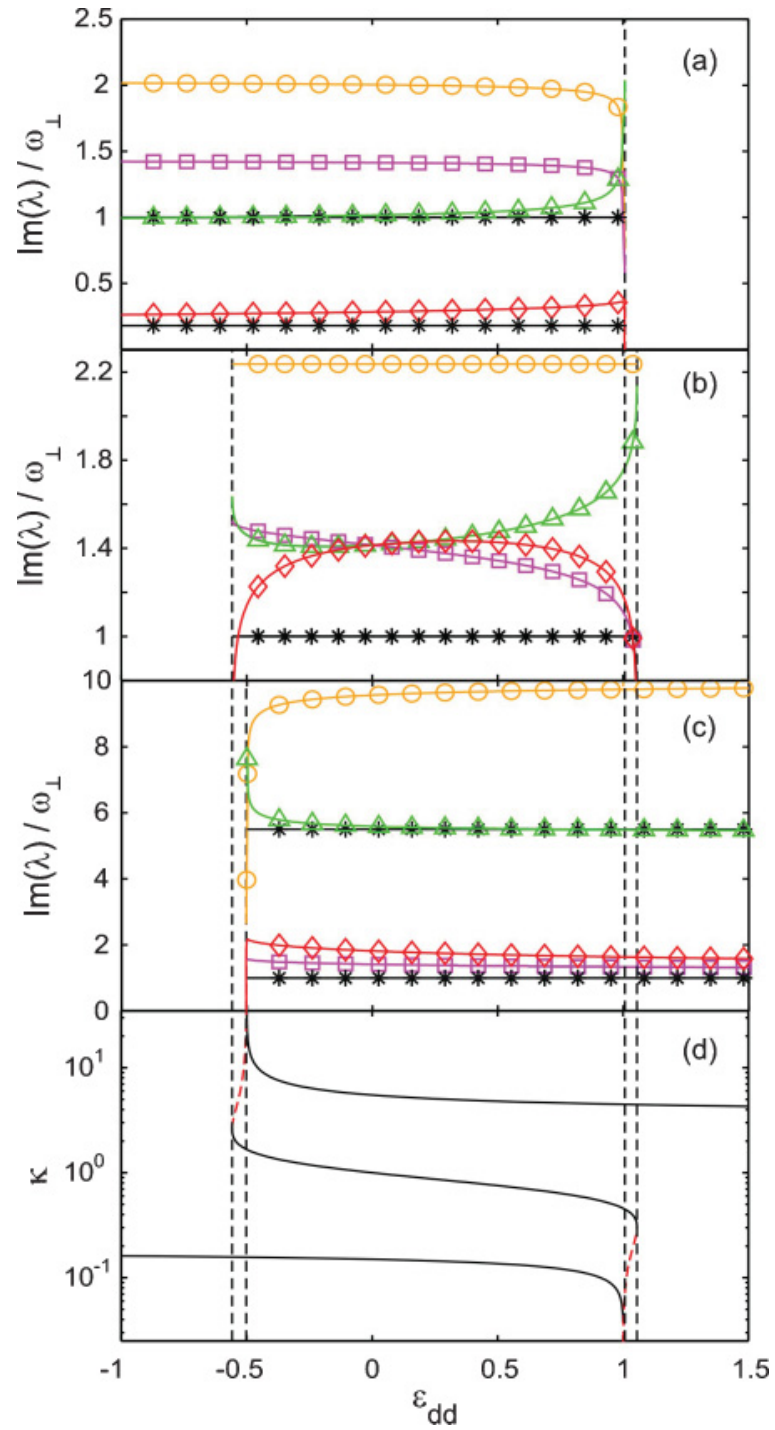

FIG. 11. (Color online) Excitation frequencies as a function of $\varepsilon_{\text {dd }}$ for a cylindrically symmetric trap with aspect ratios (a) $\gamma=0.18$, (b) $\gamma=1$, and (c) $\gamma=5.5$. Shown are the results for the dipole mode $D$ (black, stars), monopole $M$ (orange, circles), quadrupoles $Q_{1}$ (red, diamonds) and $Q_{2}$ (purple, squares), and scissors Sc (green, triangles). (d) Static solutions $\kappa$ for $\gamma=0.18$ (bottom curve), 1 (center curve), and 5.5 (top curve). Stable solutions are marked with a solid line, unstable solutions are marked with a dashed (red) line. Dashed vertical lines mark the transition point from stable to unstable.

theory, and we present the results in terms of well-known special functions (the elliptic integrals).

In the context of calculating gravitational potentials in astrophysics, one encounters exactly the same integrals as here, and as such, the problem attracted considerable interest even in the 19th century. Among others, significant contributions to the topic were made by MacLaurin, Ivory, Green, Poisson, Cayley, Ferrers, and Dyson. A detailed historical overview can be found, for instance, in Refs. [56,58]. However, for our purposes the most important contribution came from N. M. Ferrers, who showed that the potential (B1) associated with the density (B2) evaluates exactly to a polynomial in the coordinates $x, y$, and $z$ [53]. As a matter of general interest, 
we will outline the method employed by Ferrers to arrive at this remarkable result.

In his 1877 paper, Ferrers first shows how the internal (gravitational) potential of an ellipsoid with semiaxes $R_{x}, R_{y}$, and $R_{z}$, with a density of the form

$$
\rho=\rho(s)=s^{n}, \quad n=1,2,3, \ldots,
$$

with

$$
s=1-\frac{x^{2}}{R_{x}^{2}}-\frac{y^{2}}{R_{y}^{2}}-\frac{z^{2}}{R_{z}^{2}},
$$

can be calculated, using integration over so-called homoeoidal shells, to be

$$
\phi\left[s^{n}\right](x, y, z)=\frac{R_{x} R_{y} R_{z}}{4(n+1)} \int_{0}^{\infty} Q^{n+1} \frac{d \sigma}{\Delta},
$$

where

$$
Q=1-\frac{x^{2}}{R_{x}^{2}+\sigma}-\frac{y^{2}}{R_{y}^{2}+\sigma}-\frac{z^{2}}{R_{z}^{2}+\sigma},
$$

and

$$
\Delta=\sqrt{\left(R_{x}^{2}+\sigma\right)\left(R_{y}^{2}+\sigma\right)\left(R_{z}^{2}+\sigma\right)} .
$$

Homoeoidal shells are shells situated inside the ellipsoid, bounded by equidensity surfaces of the density (B3). Using infinitesimally thin homoeoidal shells, the triple integral (B1) can be reduced to a single integral over the variable $\sigma$. For a detailed account on this integration process, see, for instance, Refs. [27,55,56] or, of course, the original work by Ferrers [53]. Notably, the right-hand side of Eq. (B5) evaluates to a polynomial in $x, y$, and $z$.

Next, Ferrers noted that whenever $\rho=0$ at the boundary of the ellipsoid, then differentiation of the potential with respect to any of the Cartesian coordinates, for example, $x$, yields

$$
\frac{d}{d x} \phi[\rho](x, y, z)=\phi\left[\frac{d \rho}{d x}\right](x, y, z),
$$

which can easily be checked with integration by parts. Finally, he noted that any monomial, such as $\rho_{i j k}$ defined in Eq. (B2), can be expressed by means of a series of differential coefficients of powers $s^{m}$ of the variable $s$ defined in (B4),

$$
\rho_{i j k}=\sum_{m} \sum_{p+q+r \leqslant m} A_{m p q r}^{(i j k)} \frac{d^{p+q+r}}{d x^{p} d y^{q} d z^{r}} s^{m},
$$

where the $A_{m p q r}^{(i j k)}$ are constants, and whereby it should be noted that the order of differentiation never exceeds $m$. By virtue of the latter observation, we can calculate the potential of the above density by repeatedly applying the step (B7) in the opposite direction, transferring all differential operators appearing in (B8), from inside the integral $\phi\left[\rho_{i j k}\right]$ to the outside, since at each step we are ensured that the density being integrated over contains at least a factor of $s$, and hence is always equal to 0 on the boundary. Thus, we arrive at the following result:

$$
\begin{aligned}
\phi\left[\rho_{i j k}\right](x, y, z) & =\phi\left[\sum_{m p q r} A_{m p q r}^{(i j k)} \frac{d^{p+q+r}}{d x^{p} d y^{q} d z^{r}} s^{m}\right](x, y, z) \\
& =\sum_{m p q r} A_{m p q r}^{(i j k)} \frac{d^{p+q+r}}{d x^{p} d y^{q} d z^{r}} \phi\left[s^{m}\right](x, y, z),
\end{aligned}
$$

in which the $\phi\left[s^{m}\right]$ terms are known through Eq. (B5). Recalling that the potentials $\phi\left[s^{m}\right]$ are in fact polynomial in $x, y$, and $z$, and hence also any differential quotient, we can conclude that the potential $\phi\left[\rho_{i j k}\right]$ is also a polynomial. A crucial point in the above derivation is the observation expressed in Eq. (B8) that any monomial can be written as a series of differential coefficients of a function of the homoeoidal shell index variable $s$, which is specific to ellipsoids only. In different geometries, some monomial densities might yield polynomial potentials, but in general this is not the case.

The precise coefficients of this polynomial remain to be determined, a task undertaken by Dyson who found a compact and elegant general expression [54]. Through the efforts of Ferrers and Dyson, the triple integral of (B1) which depended on the coordinate $\mathbf{r}$, is reduced to a finite number of single integrals appearing in the coefficients of a polynomial only.

Although Dyson's formula in principle solves the problem, it is not particularly suited to numerical computation because it still contains differential operators. We therefore employ results from a more recent paper by Levin and Muratov [57], which computes the polynomial coefficients of the potential $\phi\left[\rho_{i j k}\right]$ explicitly. Levin and Muratov make use of generalized depolarization factors defined as

$$
M_{l m n}=(2 l-1) ! !(2 m-1) ! !(2 n-1) ! ! \frac{\kappa_{x} \kappa_{y} \beta_{l m n}}{2 R_{z}^{2(l+m+n-1)}},
$$

where $m, l, n=0,1,2 \ldots$, and $\beta_{l m n}$ is defined in Eq. (12). Next, we write the exponents of $\rho_{i j k}=x^{i} y^{j} z^{k}$ as

$$
i=2 \lambda+\delta_{\lambda}, \quad j=2 \mu+\delta_{\mu}, \quad k=2 v+\delta_{\nu},
$$

with $\lambda, \mu, \nu$ positive integers such that the $\delta_{\mu}, \delta_{\nu}, \delta_{\lambda}$ are either 0 or 1 for $i, j, k$ even or odd (respectively), and define

$$
\sigma=\lambda+\mu+v+1
$$

In the particular case of calculating the dipolar potential, we are interested in the second derivative with respect to $z$, rather than the potential $\phi\left[\rho_{i j k}\right]$ itself. Using the results of Levin and Muratov then, this quantity is given by

$$
\frac{\partial^{2}}{\partial z^{2}} \phi\left[\rho_{i j k}\right](x, y, z)=\frac{2 R_{x}^{i} R_{y}^{j} R_{z}^{k}}{4^{\sigma}} i ! j ! k ! \sum_{p=0}^{\sigma} \sum_{q=0}^{\sigma-p} \sum_{r=1}^{\sigma-p-q} \frac{S_{p q r}\left(2 r+\delta_{v}\right)\left(2 r+\delta_{v}-1\right) x^{2 p+\delta_{\lambda}} y^{2 q+\delta_{\mu}} z^{2 r+\delta_{v}-2}}{(\sigma-p-q-r) !\left(2 p \delta_{\lambda}+1\right)\left(2 q \delta_{\mu}+1\right)\left(2 r \delta_{v}+1\right)} \Gamma_{p q r}^{(i, j, k)},
$$

where

$$
\Gamma_{p q r}^{(i, j, k)}=\sum_{l=0}^{\lambda} \sum_{m=0}^{\mu} \sum_{n=0}^{\nu} \frac{S_{l m n} R_{x}^{2 l+\delta_{\lambda}} R_{y}^{2 m+\delta_{\mu}} R_{z}^{2 n+\delta_{v}}}{(\lambda-l) !(\mu-m) !(v-n) !\left(2 l \delta_{\lambda}+1\right)\left(2 m \delta_{\mu}+1\right)\left(2 n \delta_{v}+1\right)} M_{l+p+\delta_{\lambda}, m+q+\delta_{\mu}, n+r+\delta_{v}},
$$


and

$$
S_{l m n}=\frac{(-2)^{l+m+n}}{(2 l) !(2 m) !(2 n) !} .
$$

\section{APPENDIX C: CALCULATING THE DIPOLAR POTENTIAL INSIDE AN INVERTED PARABOLOIDAL ELLIPSOID}

In this appendix we calculate the dipolar potential inside an ellipsoidally shaped BEC with a density profile of the form

$$
n(\mathbf{r})=n_{0}\left(1-\frac{x^{2}}{R_{x}^{2}}-\frac{y^{2}}{R_{y}^{2}}-\frac{z^{2}}{R_{z}^{2}}\right)=n_{0} s,
$$

where $n_{0}$ is the density in the center of the BEC. This is a particular case of the more general density profile $\rho(\mathbf{r})=s^{n}$ discussed in Appendix B above, see Eq. (B3). Comparing with Eq. (10) in the main part of the text, we see that density profile (C1) corresponds exactly to the static solutions discussed in Sec. II. Note that the results in this appendix generalize those found in Appendix A of Ref. [27] for the cylindrically symmetric case to a triaxial ellipsoid.

In the case $n(\mathbf{r})=n_{0} s$ considered in this appendix, it is straightforward to see that the integral (B5) for the fictitious electrostatic potential $\phi(x, y, z)$ inside the ellipsoid can be expressed as

$$
\begin{aligned}
\phi= & \frac{n_{0} R_{x} R_{y} R_{z}}{2}\left(\frac{1}{4}+x^{2} \frac{\partial}{\partial\left(R_{x}^{2}\right)}+y^{2} \frac{\partial}{\partial\left(R_{y}^{2}\right)}+z^{2} \frac{\partial}{\partial\left(R_{z}^{2}\right)}\right. \\
& +2 x^{2} y^{2} \frac{\partial^{2}}{\partial\left(R_{x}^{2}\right) \partial\left(R_{y}^{2}\right)}+2 x^{2} z^{2} \frac{\partial^{2}}{\partial\left(R_{x}^{2}\right) \partial\left(R_{z}^{2}\right)} \\
& +2 y^{2} z^{2} \frac{\partial^{2}}{\partial\left(R_{y}^{2}\right) \partial\left(R_{z}^{2}\right)}+\frac{1}{3} x^{4} \frac{\partial^{2}}{\partial\left(R_{x}^{2}\right)^{2}} \\
& \left.+\frac{1}{3} y^{4} \frac{\partial^{2}}{\partial\left(R_{y}^{2}\right)^{2}}+\frac{1}{3} z^{4} \frac{\partial^{2}}{\partial\left(R_{z}^{2}\right)^{2}}\right) I_{A}\left(R_{x}, R_{y}, R_{z}\right),
\end{aligned}
$$

where

$$
I_{A}=\int_{0}^{\infty} \frac{d \sigma}{\Delta},
$$

with $\Delta$ defined in Eq. (B6). For a prolate (cigar-shaped) condensate, defined as being when $R_{z}>R_{y}>R_{x}$, we find

$$
I_{A}=\frac{2}{\sqrt{R_{z}^{2}-R_{x}^{2}}} F\left(\arccos \left[\frac{R_{x}}{R_{z}}\right] \mid \frac{R_{z}^{2}-R_{y}^{2}}{R_{z}^{2}-R_{x}^{2}}\right)
$$

where $F(\theta \mid m)$ is an elliptic integral of the first kind whose properties are well known [62]. In the opposite case of an oblate (pancake-shaped) condensate, $R_{y}>R_{x}>R_{z}$, then

$$
I_{A}=\frac{2}{\sqrt{R_{y}^{2}-R_{z}^{2}}} F\left(\arccos \left[\frac{R_{z}}{R_{y}}\right] \mid \frac{R_{y}^{2}-R_{x}^{2}}{R_{y}^{2}-R_{z}^{2}}\right) .
$$

The cylindrically symmetric case of $R_{x}=R_{y}$ is given in Appendix A of Ref. [27]. Thus, the problem of calculating the electrostatic potential $\phi(\mathbf{r})$ reduces to one of finding derivatives of elliptic integrals, both with respect to the argument $\theta$ and the parameter $m$. To evaluate the derivatives of $I_{A}$ needed in Eq. (C2) we shall make use of the results

$$
\begin{gathered}
\frac{\partial}{\partial \theta} F(\theta \mid m)=\frac{1}{\sqrt{1-m \sin ^{2} \theta}}, \\
\frac{\partial}{\partial m} F(\theta \mid m)=\frac{E(\theta \mid m)}{2 m(1-m)}-\frac{F(\theta \mid m)}{2 m} \\
-\frac{\sin 2 \theta}{4(1-m) \sqrt{1-m \sin ^{2} \theta}},
\end{gathered}
$$

where $E(\theta \mid m)$ is an elliptic integral of the second kind [62], and

$$
\begin{gathered}
\frac{\partial}{\partial \theta} E(\theta \mid m)=\sqrt{1-m \sin ^{2} \theta}, \\
\frac{\partial}{\partial m} E(\theta \mid m)=\frac{E(\theta \mid m)-F(\theta \mid m)}{2 m} .
\end{gathered}
$$

When the external polarizing field is aligned along the $z$ axis, then the mean-field dipolar potential $\Phi_{\mathrm{dd}}(\mathbf{r})$ is given by Eq. (7) as

$$
\Phi_{\mathrm{dd}}(\mathbf{r})=-C_{\mathrm{dd}}\left(\frac{\partial^{2}}{\partial z^{2}} \phi(\mathbf{r})+\frac{1}{3} n(\mathbf{r})\right) .
$$

Taking $\phi(\mathbf{r})$ from Eq. (C2), one has

$$
\begin{aligned}
\frac{\partial^{2}}{\partial z^{2}} \phi= & \frac{n_{0} R_{x} R_{y} R_{z}}{2}\left(2 \frac{\partial}{\partial\left(R_{z}^{2}\right)}+4 x^{2} \frac{\partial^{2}}{\partial\left(R_{x}^{2}\right) \partial\left(R_{z}^{2}\right)}\right. \\
& \left.+4 y^{2} \frac{\partial^{2}}{\partial\left(R_{y}^{2}\right) \partial\left(R_{z}^{2}\right)}+4 z^{2} \frac{\partial^{2}}{\partial\left(R_{z}^{2}\right)^{2}}\right) I_{A}\left(R_{x}, R_{y}, R_{z}\right) .
\end{aligned}
$$

Thus, the dipolar mean-field potential $\Phi_{\mathrm{dd}}(\mathbf{r})$ inside the inverted parabola density profile $(\mathrm{C} 1)$ is itself a quadratic function of position $(x, y, z)$, as given in Eq. (11),

$$
\begin{aligned}
\Phi_{\mathrm{dd}}(\mathbf{r})= & -g \varepsilon_{\mathrm{dd}} n(\mathbf{r})+\frac{3 g \varepsilon_{\mathrm{dd}} n_{0} \kappa_{x} \kappa_{y}}{2} \\
& \times\left[\beta_{001}-\left(\beta_{101} x^{2}+\beta_{011} y^{2}+3 \beta_{002} z^{2}\right) R_{z}^{-2}\right],
\end{aligned}
$$

where $\kappa_{i} \equiv R_{i} / R_{z}$, and the coefficients $\beta_{i j k}$ defined in Eq. (12) can be seen to be

$$
\begin{gathered}
\beta_{001}=-2 R_{z}^{3} \frac{\partial}{\partial\left(R_{z}^{2}\right)} I_{A}\left(R_{x}, R_{y}, R_{z}\right), \\
\beta_{101}=4 R_{z}^{5} \frac{\partial^{2}}{\partial\left(R_{x}^{2}\right)\left(R_{z}^{2}\right)} I_{A}\left(R_{x}, R_{y}, R_{z}\right), \\
\beta_{011}=4 R_{z}^{5} \frac{\partial^{2}}{\partial\left(R_{y}^{2}\right)\left(R_{z}^{2}\right)} I_{A}\left(R_{x}, R_{y}, R_{z}\right), \\
\beta_{002}=\frac{4}{3} R_{z}^{5} \frac{\partial^{2}}{\partial\left(R_{z}^{2}\right)^{2}} I_{A}\left(R_{x}, R_{y}, R_{z}\right) .
\end{gathered}
$$

Using (C6)-(C9) to perform the required derivatives, an explicit expression for the dipolar mean-field potential $\Phi_{\mathrm{dd}}$ 
can be given in terms of elliptic integrals. In the prolate case, when $R_{z}>R_{y}>R_{x}$, then

$$
\begin{aligned}
\beta_{001}= & -\frac{2}{\sqrt{1-\kappa_{x}^{2}}\left(1-\kappa_{y}^{2}\right)}\left\{E\left(\arccos \left[\kappa_{x}\right] \mid \frac{1-\kappa_{y}^{2}}{1-\kappa_{x}^{2}}\right)-F\left(\arccos \left[\kappa_{x}\right] \mid \frac{1-\kappa_{y}^{2}}{1-\kappa_{x}^{2}}\right)\right\}, \\
\beta_{101}= & 2\left\{\frac{\kappa_{y}}{\kappa_{x}} \frac{1+\kappa_{x}^{2}-2 \kappa_{y}^{2}}{\left(1-\kappa_{x}^{2}\right)\left(\kappa_{y}^{2}-\kappa_{x}^{2}\right)}-\frac{1}{\left(1-\kappa_{x}^{2}\right)^{3 / 2}\left(\kappa_{y}^{2}-\kappa_{x}^{2}\right)\left(1-\kappa_{y}^{2}\right)} E\left(\arccos \left[\kappa_{x}\right] \mid \frac{1-\kappa_{y}^{2}}{1-\kappa_{x}^{2}}\right)\right. \\
& \left.-\frac{1}{\left(1-\kappa_{x}^{2}\right)^{3 / 2}\left(1-\kappa_{y}^{2}\right)} F\left(\arccos \left[\kappa_{x}\right] \mid \frac{1-\kappa_{y}^{2}}{1-\kappa_{x}^{2}}\right)\right\}, \\
\beta_{011}= & \left\{-\frac{\kappa_{x}}{\kappa_{y}} \frac{1-2 \kappa_{x}^{2}+\kappa_{y}^{2}}{\left(1-\kappa_{y}^{2}\right)\left(\kappa_{y}^{2}-\kappa_{x}^{2}\right)}+\frac{2}{\sqrt{1-\kappa_{x}^{2}}\left(\kappa_{y}^{2}-\kappa_{x}^{2}\right)\left(1-\kappa_{y}^{2}\right)^{2}} E\left(\arccos \left[\kappa_{x}\right] \mid \frac{1-\kappa_{y}^{2}}{1-\kappa_{x}^{2}}\right)\right. \\
& \left.-\frac{2}{\sqrt{1-\kappa_{x}^{2}}\left(1-\kappa_{y}^{2}\right)^{2}} F\left(\arccos \left[\kappa_{x}\right] \mid \frac{1-\kappa_{y}^{2}}{1-\kappa_{x}^{2}}\right)\right\}, \\
\beta_{002}= & \frac{2}{3}\left\{\frac{\kappa_{x} \kappa_{y}}{\left(1-\kappa_{y}^{2}\right)\left(1-\kappa_{x}^{2}\right)}-\frac{2\left(2-\kappa_{x}^{2}-\kappa_{y}^{2}\right)}{\left(1-\kappa_{x}^{2}\right)^{3 / 2}\left(1-\kappa_{y}^{2}\right)^{2}} E\left(\arccos \left[\kappa_{x}\right] \mid \frac{1-\kappa_{y}^{2}}{1-\kappa_{x}^{2}}\right)\right. \\
& \left.+\frac{\left(3-2 \kappa_{x}^{2}-\kappa_{y}^{2}\right)}{\left(1-\kappa_{x}^{2}\right)^{3 / 2}\left(1-\kappa_{y}^{2}\right)^{2}} F\left(\arccos \left[\kappa_{x}\right] \mid \frac{1-\kappa_{y}^{2}}{1-\kappa_{x}^{2}}\right)\right\} .
\end{aligned}
$$

While in the oblate case, when $R_{y}>R_{x}>R_{z}$, we have

$$
\begin{aligned}
& \beta_{001}=-\frac{2}{\left(1-\kappa_{x}^{2}\right)\left(1-\kappa_{y}^{2}\right) \kappa_{y}}\left\{\kappa_{x}\left(1-\kappa_{y}^{2}\right)+\kappa_{y} \sqrt{\kappa_{y}^{2}-1} E\left(\arccos \left[\frac{1}{\kappa_{y}}\right] \mid \frac{\kappa_{y}^{2}-\kappa_{x}^{2}}{\kappa_{y}^{2}-1}\right)\right\}, \\
& \beta_{101}=2\left\{\frac{1}{\kappa_{x} \kappa_{y}} \frac{1+\kappa_{x}^{2}}{\left(1-\kappa_{x}^{2}\right)^{2}}+\frac{\kappa_{x}^{2}-2 \kappa_{y}^{2}+1}{\left(\kappa_{y}^{2}-\kappa_{x}^{2}\right)\left(\kappa_{x}^{2}-1\right)^{2} \sqrt{\kappa_{y}^{2}-1}} E\left(\arccos \left[\frac{1}{\kappa_{y}}\right] \mid \frac{\kappa_{y}^{2}-\kappa_{x}^{2}}{\kappa_{y}^{2}-1}\right)\right. \\
& \left.+\frac{1}{\left(\kappa_{y}^{2}-\kappa_{x}^{2}\right)\left(\kappa_{x}^{2}-1\right) \sqrt{\kappa_{y}^{2}-1}} F\left(\arccos \left[\frac{1}{\kappa_{y}}\right] \mid \frac{\kappa_{y}^{2}-\kappa_{x}^{2}}{\kappa_{y}^{2}-1}\right)\right\}, \\
& \beta_{011}=2\left\{\frac{\kappa_{x}}{\kappa_{y}} \frac{1}{\left(\kappa_{x}^{2}-1\right)\left(\kappa_{y}^{2}-1\right)}+\frac{2 \kappa_{x}^{2}-\kappa_{y}^{2}-1}{\left(\kappa_{y}^{2}-\kappa_{x}^{2}\right)\left(\kappa_{x}^{2}-1\right)\left(\kappa_{y}^{2}-1\right)^{3 / 2}} E\left(\arccos \left[\frac{1}{\kappa_{y}}\right] \mid \frac{\kappa_{y}^{2}-\kappa_{x}^{2}}{\kappa_{y}^{2}-1}\right)\right. \\
& \left.-\frac{1}{\left(\kappa_{y}^{2}-\kappa_{x}^{2}\right)\left(\kappa_{y}^{2}-1\right)^{3 / 2}} F\left(\arccos \left[\frac{1}{\kappa_{y}}\right] \mid \frac{\kappa_{y}^{2}-\kappa_{x}^{2}}{\kappa_{y}^{2}-1}\right)\right\} \text {, } \\
& \beta_{002}=\frac{2}{3}\left\{\frac{\kappa_{x}}{\kappa_{y}} \frac{\left(\kappa_{x}^{2} \kappa_{y}^{2}-2 \kappa_{x}^{2}-3 \kappa_{y}^{2}+4\right)}{\left(\kappa_{x}^{2}-1\right)^{2}\left(\kappa_{y}^{2}-1\right)}+\frac{2\left(\kappa_{x}^{2}+\kappa_{y}^{2}-2\right)}{\left(\kappa_{x}^{2}-1\right)^{2}\left(\kappa_{y}^{2}-1\right)^{3 / 2}} E\left(\arccos \left[\frac{1}{\kappa_{y}}\right] \mid \frac{\kappa_{y}^{2}-\kappa_{x}^{2}}{\kappa_{y}^{2}-1}\right)\right. \\
& \left.-\frac{1}{\left(\kappa_{x}^{2}-1\right)\left(\kappa_{y}^{2}-1\right)^{3 / 2}} F\left(\arccos \left[\frac{1}{\kappa_{y}}\right] \mid \frac{\kappa_{y}^{2}-\kappa_{x}^{2}}{\kappa_{y}^{2}-1}\right)\right\} \text {. }
\end{aligned}
$$


[1] M. H. Anderson, J. R. Ensher, M. R. Matthews, C. E. Wieman, and E. A. Cornell, Science 269, 198 (1995); K. B. Davis, M. O. Mewes, M. R. Andrews, N. J. van Druten, D. S. Durfee, D. M. Kurn, and W. Ketterle, Phys. Rev. Lett. 75, 3969 (1995); C. C. Bradley, C. A. Sackett, J. J. Tollett, and R. G. Hulet, ibid. 75, 1687 (1995).

[2] C. J. Pethick and H. Smith, Bose-Einstein Condensation in Dilute Gases (Cambridge University Press, Cambridge, England, 2001).

[3] L. Pitaevskii and S. Stringari, Bose-Einstein Condensation (Clarendon, Oxford, 2003).

[4] A. Griesmaier, J. Werner, S. Hensler, J. Stuhler, and T. Pfau, Phys. Rev. Lett. 94, 160401 (2005).

[5] Q. Beaufils, R. Chicireanu, T. Zanon, B. Laburthe-Tolra, E. Maréchal, L. Vernac, J.-C. Keller, and O. Gorceix, Phys. Rev. A 77, 061601(R) (2008).

[6] M. Fattori, G. Roati, B. Deissler, C. D'Errico, M. Zaccanti, M. Jona-Lasinio, L. Santos, M. Inguscio, and G. Modugno, Phys. Rev. Lett. 101, 190405 (2008).

[7] M. Vengalattore, S. R. Leslie, J. Guzman, and D. M. StamperKurn, Phys. Rev. Lett. 100, 170403 (2008).

[8] J. Stuhler, A. Griesmaier, T. Koch, M. Fattori, T. Pfau, S. Giovanazzi, P. Pedri, and L. Santos, Phys. Rev. Lett. 95, 150406 (2005).

[9] T. Lahaye, T. Koch, B. Fröhlich, M. Fattori, J. Metz, A. Griesmaier, S. Giovanazzi, and T. Pfau, Nature (London) 448, 672 (2007).

[10] T. Lahaye, J. Metz, B. Fröhlich, T. Koch, M. Meister, A. Griesmaier, T. Pfau, H. Saito, Y. Kawaguchi, and M. Ueda, Phys. Rev. Lett. 101, 080401 (2008).

[11] T. Koch, T. Lahaye, J. Metz, B. Fröhlich, A. Griesmaier, and T. Pfau, Nature Phys. 4, 218 (2008).

[12] K. Góral, K. Rzazewski, and T. Pfau, Phys. Rev. A 61, 051601(R) (2000).

[13] L. Santos, G. V. Shlyapnikov, P. Zoller, and M. Lewenstein, Phys. Rev. Lett. 85, 1791 (2000).

[14] S. Yi and L. You, Phys. Rev. A 63, 053607 (2001).

[15] K. Kanjilal, J. L. Bohn, and D. Blume, Phys. Rev. A 75, 052705 (2007).

[16] S. Giovanazzi, A. Görlitz, and T. Pfau, Phys. Rev. Lett. 89, 130401 (2002).

[17] H. Feshbach, Ann. Phys. (NY) 5, 357 (1958); 19, 287 (1962).

[18] J. Werner, A. Griesmaier, S. Hensler, J. Stuhler, T. Pfau, A. Simoni, and E. Tiesinga, Phys. Rev. Lett. 94, 183201 (2005).

[19] J. Doyle, B. Friedrich, R. V. Krems, and F. Masnou-Seeuws, Eur. Phys. J. D 31, 149 (2004).

[20] J. M. Sage, S. Sainis, T. Bergeman, and D. DeMille, Phys. Rev. Lett. 94, 203001 (2005).

[21] T. Köhler, K. Góral, and P. S. Julienne, Rev. Mod. Phys. 78, 1311 (2006).

[22] C. Ospelkaus, S. Ospelkaus, L. Humbert, P. Ernst, K. Sengstock, and K. Bongs, Phys. Rev. Lett. 97, 120402 (2006).

[23] K.-K. Ni et al., Science 322, 231 (2008).

[24] K.-K. Ni, S. Ospelkaus, D. Wang, G. Quéméner, B. Neyenhuis, M. H. G. de Miranda, J. L. Bohn, J. Ye, and D. S. Jin, Nature 464, 1324 (2010).

[25] K. Góral and L. Santos, Phys. Rev. A 66, 023613 (2002).

[26] D. H. J. O'Dell, S. Giovanazzi, and C. Eberlein, Phys. Rev. Lett. 92, 250401 (2004).
[27] C. Eberlein, S. Giovanazzi, and D. H. J. O’Dell, Phys. Rev. A 71, 033618 (2005).

[28] S. Ronen, D. C. E. Bortolotti, and J. L. Bohn, Phys. Rev. A 74, 013623 (2006).

[29] S. Ronen, D. C. E. Bortolotti, and J. L. Bohn, Phys. Rev. Lett. 98, 030406 (2007).

[30] O. Dutta and P. Meystre, Phys. Rev. A 75, 053604 (2007).

[31] O. Dutta, R. Kanamoto, and P. Meystre, Phys. Rev. A 78, 043608 (2008).

[32] N. G. Parker, C. Ticknor, A. M. Martin, and D. H. J. O'Dell, Phys. Rev. A 79, 013617 (2009).

[33] S. Stringari, Phys. Rev. Lett. 77, 2360 (1996).

[34] N. G. Parker and D. H. J. O’Dell, Phys. Rev. A 78, 041601(R) (2008).

[35] R. M. W. van Bijnen, D. H. J. O’Dell, N. G. Parker, and A. M. Martin, Phys. Rev. Lett. 98, 150401 (2007).

[36] R. M. W. van Bijnen, A. J. Dow, D. H. J. O’Dell, N. G. Parker, and A. M. Martin, Phys. Rev. A 80, 033617 (2009).

[37] D. M. Harber, J. M. Obrecht, J. M. McGuirk, and E. A. Cornell, Phys. Rev. A 72, 033610 (2005); J. M. Obrecht, R. J. Wild, M. Antezza, L. P. Pitaevskii, S. Stringari, and E. A. Cornell, Phys. Rev. Lett. 98, 063201 (2007).

[38] D. Guèry-Odelin and S. Stringari, Phys. Rev. Lett. 83, 4452 (1999).

[39] O. M. Maragò, S. A. Hopkins, J. Arlt, E. Hodby, G. Hechenblaikner, and C. J. Foot, Phys. Rev. Lett. 84, 2056 (2000).

[40] F. Zambelli and S. Stringari, Phys. Rev. A 63, 033602 (2001).

[41] M. Cozzini, S. Stringari, V. Bretin, P. Rosenbusch, and J. Dalibard, Phys. Rev. A 67, 021602(R) (2003).

[42] S. Sinha and Y. Castin, Phys. Rev. Lett. 87, 190402 (2001).

[43] N. G. Parker, R. M. W. van Bijnen, and A. M. Martin, Phys. Rev. A 73, 061603(R) (2006).

[44] A. Recati, F. Zambelli, and S. Stringari, Phys. Rev. Lett. 86, 377 (2001).

[45] K. W. Madison, F. Chevy, V. Bretin, and J. Dalibard, Phys. Rev. Lett. 86, 4443 (2001).

[46] I. Corro, N. G. Parker, and A. M. Martin, J. Phys. B 40, 3615 (2007)

[47] C. Ticknor, N. G. Parker, A. Melatos, S. L. Cornish, D. H. J. O'Dell, and A. M. Martin, Phys. Rev. A 78, 061607(R) (2008).

[48] S. Yi and L. You, Phys. Rev. A 66, 013607 (2002).

[49] D. H. J. O’Dell, S. Giovanazzi, and G. Kurizki, Phys. Rev. Lett. 90, 110402 (2003); S. Giovanazzi and D. H. J. O’Dell, Eur. Phys. J. D 31, 439 (2004).

[50] L. Santos, G. V. Shlyapnikov, and M. Lewenstein, Phys. Rev. Lett. 90, 250403 (2003).

[51] S. Giovanazzi, L. Santos, and T. Pfau, Phys. Rev. A 75, 015604 (2007).

[52] See supplementary material at [http://link.aps.org/supplemental/ 10.1103/PhysRevA.82.033612], for a MATLAB implementation of the excitation frequency calculations with a graphical user interface.

[53] N. M. Ferrers, Q. J. Pure Appl. Math. 14, 1 (1877).

[54] F. W. Dyson, Q. J. Pure Appl. Math. 25, 259 (1891).

[55] E. J. Routh, A Treatise on Analytical Statics (Cambridge University Press, Cambridge, England, 1892), Vol. II.

[56] S. Chandrasekhar, Ellipsoidal Figures of Equilibrium (Dover, New York, 1988).

[57] M. L. Levin and R. Z. Muratov, Astrophys. J. 166, 441 (1971). 
[58] M. Rahman, Proc. R. Soc. London A 457, 2227 (2001).

[59] D. P. Craig and T. Thirunamachandran, Molecular Quantum Electrodynamics (Academic, London, 1984), p. 56.

[60] J. H. Hannay, Eur. J. Phys. 4, 141 (1983).

[61] Table of Integrals, Series, and Products, 6th ed., edited by L. S. Gradshteyn and I. M. Ryzhik (Academic, San Diego, 2000).

[62] Handbook of Mathematical Functions, edited by M. Abramowitz and I. Stegun (Dover, New York, 1974).

[63] Yu. Kagan, E. L. Surkov, and G. V. Shlyapnikov, Phys. Rev. A 54, R1753 (1996); 55, R18 (1997).

[64] Y. Castin and R. Dum, Phys. Rev. Lett. 77, 5315 (1996).

[65] C. A. Sackett, J. M. Gerton, M. Welling, and R. G. Hulet, Phys. Rev. Lett. 82, 876 (1999); J. M. Gerton, D. Strekalov, I. Prodan, and R. G. Hulet, Nature (London) 408, 692 (2000); J. L. Roberts, N. R. Claussen, S. L. Cornish, E. A. Donley, E. A. Cornell, and C. E. Wieman, Phys. Rev. Lett. 86, 4211 (2001).

[66] P. A. Ruprecht, M. J. Holland, K. Burnett, and M. Edwards, Phys. Rev. A 51, 4704 (1995).

[67] C. A. Sackett, H. T. C. Stoof, and R. G. Hulet, Phys. Rev. Lett. 80, 2031 (1998).

[68] V. M. Pérez-García, H. Michinel, J. I. Cirac, M. Lewenstein, and P. Zoller, Phys. Rev. Lett. 77, 5320 (1996).

[69] U. Al Khawaja and H. T. C. Stoof, Phys. Rev. A 65, 013605 (2001).
[70] In some of our preceding work [34,35,42], the prefactors of the $\left(1+\mathcal{K} \varepsilon_{\mathrm{dd}}\right)$ and $\nabla \cdot n_{\mathrm{eq}} \nabla$ terms appearing in Eq. (25) were misquoted (although the ensuing results were unaffected) and these terms are now in their correct form.

[71] For rotating systems, the directions of oscillation will differ from the trap axes, but these systems are not considered in this work.

[72] D. H. J. O'Dell and C. Eberlein, Phys. Rev. A 75, 013604 (2007).

[73] Emergent Nonlinear Phenomena in Bose-Einstein Condensates: Theory and Experiment, edited by P. G. Kevrekidis, D. J. Frantzeskakis, and R. Carretero-González, Springer Series on Atomic, Optical, and Plasma Physics Vol. 45 (Springer, Berlin, 2009).

[74] K. Huang, Statistical Mechanics, 2nd ed. (Wiley, New York, 1987).

[75] P. Nozières in Bose Einstein Condensation, edited by A. Griffin, D. W. Snoke, and S. Stringari (Cambridge University Press, New York, 1995), p. 15.

[76] A. J. Leggett, Rev. Mod. Phys. 73, 307 (2001).

[77] E. J. Mueller, T.-L. Ho, M. Ueda, and G. Baym, Phys. Rev. A 74, 033612 (2006).

[78] P. Bader and U. R. Fischer, Phys. Rev. Lett. 103, 060402 (2009); U. R. Fischer and P. Bader, Phys. Rev. A 82, 013607 (2010).

[79] G. Bismut et al., Phys. Rev. Lett. 105, 040404 (2010). 